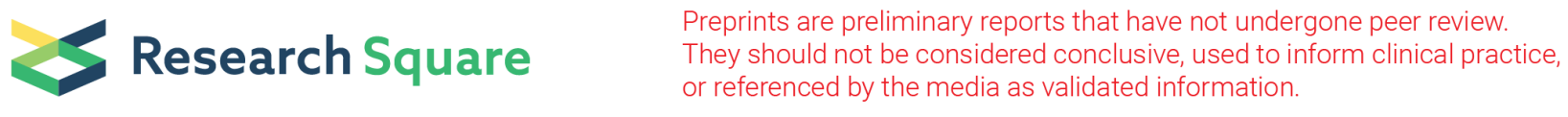

\title{
Title: The Effectiveness of Participant Blinding of Non-Penetrating Sham/Placebo Acupuncture in Clinical Trials: A Systematic Review with Meta- Analysis
}

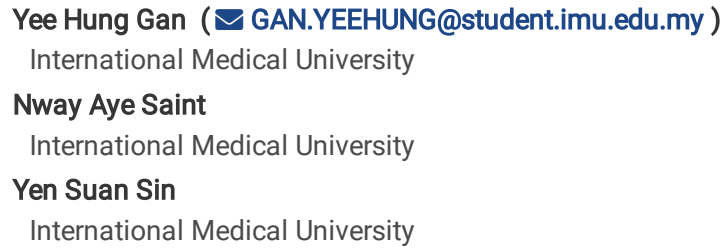

\section{Research Article}

Keywords: Sham acupuncture, Placebo acupuncture, Participant blinding, Non-penetrating, Systematic review, Meta-analysis

Posted Date: February 17th, 2021

DOI: https://doi.org/10.21203/rs.3.rs-156277/v1

License: (c) (i) This work is licensed under a Creative Commons Attribution 4.0 International License. Read Full License 


\section{Abstract}

Background: Acupuncture clinical trial is important to evaluate the efficacy of acupuncture. However, it is challenging to achieve effective blinding due to the nature of acupuncture. A standardised placebo control method of acupuncture has yet to be established. The study focuses on the non-penetrating sham acupuncture because it eliminates the placebo effect and generates lesser physiological responses. The study aims to evaluate and compare the participant blinding effectiveness of non-penetrating sham acupuncture devices, and analyse the factors which may influence the participant blinding.

Methods: The study followed the PRISMA guidelines. An electronic search was conducted on PubMed, Ovid and CNKI up until 1st of October 2020 to include English and Chinese randomised controlled trials which evaluated the awareness on the type of acupuncture (real or sham) in any population who received acupuncture. Data screening, data extraction and quality assessment were done independently by two researchers and discrepancies were sorted out via discussion with a co-researcher. Data analysis was performed using RevMan 5.4.1.

Results: 34 full-text articles had been included in the systematic review and meta-analysis. The quality of the studies ranged from moderate to good. Generally, non-penetrating sham acupuncture devices were effective in blinding participants in clinical trials. The foam device demonstrated a better blinding effect, followed by Streitberger, Park and Takakura devices. Sham needles with no skin contact could not blind the participants successfully. Naive, experienced, healthy and diseased participants all could be blinded using non-penetrating sham acupuncture devices but naive and healthy participants could be blinded comparatively easily. Acupoints from different regions could achieve blinding, however, the acupoints on the back could blind the participants more easily compared to the other areas.

Conclusion: Non-penetrating sham acupuncture devices are valid placebo control for acupuncture clinical trials. The foam device has a better blinding effect, followed by Streitberger, Park and Takakura devices. Recruiting naive healthy participants and choosing acupoints from the back can achieve better blinding effects in the participants.

\section{Background}

Acupuncture has been popular in Asia, serving as one of the major treatment methods for thousands of years. In the sixteenth century, it had been introduced to Europe and America. After that, it gradually spread all around the world and gained popularity due to its therapeutic effects, including analgesic, anaesthetic, mind-calming and body regulating effects. To investigate its clinical efficacy, acupuncture research was initiated in the eighteenth century and conducted continuously since then ${ }^{1}$.

A well-blinded randomised controlled trial (RCT) is the gold standard of clinical trials because it minimises the risk of bias and maximises the validity of the results $^{2-4}$. In an ideal acupuncture clinical trial, subjects should be randomly assigned to a treatment or control group, and both the participants and acupuncturists should be blinded. It is challenging to achieve effective blinding in acupuncture clinical trials due to acupuncture's nature, whereby the participants can feel the needling sensation and deduce the grouping, whereas the acupuncturists are not blinded most of the time because they need to know where and how to needle the participants, and can also feel the penetrating sensation from their fingers.

There are several types of control groups that are commonly used, such as no treatment, standard/conventional treatment, sham/placebo acupuncture and minimal acupuncture (shallow needle insertion) ${ }^{4}$. Non-treatment or standard/conventional treatment as the control cannot eliminate placebo effects. Moreover, skin penetrating sham acupuncture as the control may trigger physiological responses. For instance, it can deactivate limbic structures and, hence, reduce pain levels in patients who suffer from pain ${ }^{5-6}$. Thus, to assess the true efficacy of acupuncture, selecting a good non-penetrating sham/placebo method is necessary.

Currently, a standardised sham/placebo acupuncture method has yet to be established. Various types of non-penetrating sham devices have been developed to raise the quality of clinical trials and the validity of the results, including Streitberger device ${ }^{7}$, Park device ${ }^{8}$, Takakura device ${ }^{9}$ and foam device ${ }^{10}$. Many studies tried to evaluate the blinding effectiveness of individual sham devices but the sample size was small for most of the studies. There was insufficient evidence to demonstrate the superiority of a specific type of sham device in achieving participants' blinding. Therefore, there is a need to perform a systematic review with meta-analysis to evaluate the blinding effectiveness of these sham acupuncture devices.

Zhang et $\mathrm{al}^{11}$ conducted a systematic review on the credibility of blinding healthy participants and/or acupuncturists using placebo acupuncture devices. The author also studied the penetrating sensation and DeQi sensation of the devices. The study found that the real and sham Streitberger and Park devices were significantly different in penetrating sensation when applied at sensitive acupuncture points, which may lead to failure in participants' blinding. However, there was no meta-analysis performed to compare the blinding effectiveness of different sham acupuncture devices. The study only included the results in phase one which could lead to an incomplete assessment of the outcome, especially for cross-over studies.

Gong et al ${ }^{12}$ carried out a systematic review with meta-analysis on the blinding effectiveness of non-penetrating sham needles. The study included five articles and found that participants were not able to differentiate between real and sham acupuncture. The author pointed out that it may be necessary to analyse the influence of age, sex, ethnic admixture and other factors. The number of included studies was too small to perform a meaningful comparison among devices.

In short, a limited number of studies had systematically reviewed and analysed the blinding effectiveness of sham acupuncture devices. A comparison among sham acupuncture devices was not performed to show the superiority of a specific sham device in blinding participants. Factors which may influence the blinding results, for example, acupuncture experience and health status of participants, and needling locations, were not statistically analysed in the existing

Page $2 / 20$ 
studies. As a result, this study aims to assess and compare the blinding effectiveness of non-penetrating sham acupuncture devices as well as analyse the factors which may influence the blinding effectiveness.

\section{Methods}

This systematic review and meta-analysis followed the Preferred Reporting Items for Systematic Reviews and Meta-Analysis Statement (PRISMA) guidelines ${ }^{13}$.

\section{Search Strategy}

An electronic search was carried out in health-related databases such as PubMed, Ovid and CNKI for relevant studies. The literature search was conducted using search terms such as sham acupuncture, placebo acupuncture, sham needle, placebo needle, Park needle, Park device, Streitberger needle, Streitberger

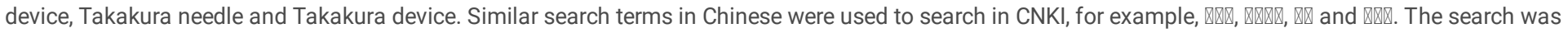
limited to original articles published in English and Chinese language until 1st October 2020. Additional studies were identified manually from reference lists of potentially eligible articles. Title and abstract of those studies were screened to determine its relevance.

\section{Inclusion Criteria}

Selection of primary studies for this review was derived from the following pre-specified criteria (in PICOS format). Type of participants (P)Any population who received acupuncture treatment regardless of age, sex, race, region, underlying disease and acupuncture experiencelntervention (I)Non-penetrating sham/placebo acupunctureComparison (C)Real penetrating acupunctureOutcomes (0)Awareness of participants on the type of acupunctureType of studies (S)Randomised Controlled Trials (RCTs)

\section{Exclusion Criteria}

Studies were excluded if the studies were animal studies, review articles, case reports, editorials, letters and comments. Studies published other than English and Chinese were excluded. Studies which did not meet any of the inclusion criteria were also excluded.

\section{Data Extraction}

Data were extracted by using a review spreadsheet, containing information such as author, publication year, study design (blinding and sample size), participant details (age, gender, health status and acupuncture experience), acupuncture methods (the type of sham acupuncture device and needling location) and results. Corresponding authors were contacted if the data was unclear. The data extraction was conducted separately by two researchers. Any discrepancies were sorted out via discussion with a co-researcher.

\section{Quality Assessment}

Quality and risk of bias of the eligible studies were assessed independently by two researchers at outcome level by using Cochrane risk of bias tool ${ }^{14}$. Quality assessments included random sequence generation, allocation concealment, blinding of participants and personnel, blinding of outcome assessment, incomplete outcome data, selective reporting and other items. Studies were graded as low, unclear or high risk of bias. Discrepancies were resolved via discussion among the researchers. Publication bias across the studies was presented as funnel plot.

\section{Data Analysis}

Meta-analysis was performed using RevMan 5.4.1 ${ }^{15}$. The effectiveness of blinding in sham/placebo acupuncture compared to real acupuncture was estimated with the odds ratios (OR) and its $95 \%$ confidence intervals. OR > 1 means blinding is more likely to occur in the intervention arm (sham group) than in the comparator arm (real group). Trials which the patients had no events in both intervention and comparator arms were excluded from the meta-analysis. Heterogeneities were assessed using the chi-squared $\left(\mathrm{X}^{2}\right)$ test and the inconsistency index $\left(\mathrm{I}^{2}\right)$ statistic. A two-tailed $\mathrm{P}$ value of less than 0.05 was considered as statistically significant. Subgroup analyses were performed on the factors which could influence the blinding effectiveness of participants.

\section{Results}

\section{Study Selection}

A total number of 1189 studies were identified from the databases and 18 studies from the reference list of related studies (Fig. 1). After screening, 34 studies had been included in the systematic review and meta-analysis. 28 studies ${ }^{16-43}$ had been excluded with reasons during the screening of full-text articles (Table 1). 
Table 1

Excluded Studies with Reasons

\begin{tabular}{|c|c|}
\hline Excluded Studies & Reasons \\
\hline Chae $2006^{16}$ & The study published in Korean \\
\hline Dilli $2013^{17}$ & Not a randomised controlled trial \\
\hline Fink $2004^{18}$ & No reported data on the awareness of the device used \\
\hline Fink $2005^{19}$ & No reported data on the awareness of the device used \\
\hline Foster $2007^{20}$ & No reported data on the awareness of the device used \\
\hline Francia $2018^{21}$ & Focused on the blinding of acupuncturist only \\
\hline Grillo $2018^{22}$ & No reported data on the awareness of the device used \\
\hline Hu $2020^{23}$ & Not a randomised controlled trial \\
\hline Jiang $2014^{24}$ & Inaccessible full-text article and no reply from the author \\
\hline Lee $2006^{25}$ & Inaccessible full-text article and no reply from the author \\
\hline Leem $2016^{26}$ & No reported data on the awareness of the device used \\
\hline $\operatorname{Lim} 2006^{27}$ & The study published in Korean \\
\hline Park $2000^{28}$ & The study published in Japanese \\
\hline Park $2002^{29}$ & Inaccessible full-text article and no reply from the author \\
\hline Park $2008^{30}$ & The study published in Korean \\
\hline Park $2009^{31}$ & Not a randomised controlled trial \\
\hline Park $2010^{32}$ & Inaccessible full-text article and no reply from the author \\
\hline Streitberger $2003^{33}$ & No reported data on the awareness of the device used \\
\hline Streitberger $2004^{34}$ & No reported data on the awareness of the device used \\
\hline Takakura $2010^{35}$ & Focused on the blinding of acupuncturist only \\
\hline Takakura $2013^{36}$ & Inaccessible full-text article and no reply from the author \\
\hline Takakura $2014^{37}$ & No reported data on the awareness of the device used \\
\hline Tsukayama $2005^{38}$ & Inaccessible full-text article and no reply from the author \\
\hline Vickers $2005^{39}$ & Insufficient data on the awareness of the device used \\
\hline White $2000^{40}$ & Insufficient data on the awareness of the device used \\
\hline Wong $2018^{41}$ & Insufficient data on the awareness of the device used \\
\hline Yan $2016^{42}$ & No reported data on the awareness of the device used \\
\hline Zaslawski $1997^{43}$ & Inaccessible full-text article and no reply from the author \\
\hline
\end{tabular}

\section{Study Characteristics}

The characteristics of all 34 included studies ${ }^{7-10,44-73}$ had been summarised in Table 2. The publication year of recruited studies ranged from 1996 to 2019. All the studies performed participant blinding, but only some of them tried to blind acupuncturist $9,10,52,59-62,66,69$, outcome assessor $8,47,51,53,56,60,70$ or statistician $50,55,56$. The number of participants recruited in these studies ranged from 10 to 321 . The total number of participants involved was 2538 . 
Table 2

Characteristics of the Included Studies

\begin{tabular}{|c|c|c|c|c|c|c|c|c|c|c|}
\hline \multirow[t]{2}{*}{ Study ID } & \multicolumn{2}{|l|}{ Study Design } & \multicolumn{3}{|l|}{ Participants } & \multicolumn{3}{|c|}{ Needling } & \multicolumn{2}{|c|}{$\begin{array}{l}\text { Awareness of thi } \\
\text { of Acupuncture }\end{array}$} \\
\hline & Blinding & $\begin{array}{l}\text { Sample } \\
\text { Size }\end{array}$ & $\begin{array}{l}\text { Mean/Median } \\
\text { Age (Range) } \\
\text { in Years }\end{array}$ & $\begin{array}{l}\text { Gender } \\
(\mathrm{M}: \mathrm{F})\end{array}$ & Health Status & $\begin{array}{l}\text { Acupuncture } \\
\text { Experience }\end{array}$ & $\begin{array}{l}\text { Types of } \\
\text { Sham } \\
\text { Device }\end{array}$ & Location & Real & She \\
\hline $\begin{array}{l}\text { Chae } \\
2010^{44}\end{array}$ & Participants & 14 & $(22-35)$ & $8: 6$ & Healthy & Experienced & Park & LI4 & $11 / 14^{\wedge}$ & $12 /$ \\
\hline $\begin{array}{l}\text { Chen } \\
2005^{45}\end{array}$ & Participants & 60 & $(21-40)$ & $31: 29$ & Healthy & Naive & Streitberger & LI4 & $54 / 60^{\wedge}$ & $13 /$ \\
\hline $\begin{array}{l}\text { Deng } \\
2007^{46}\end{array}$ & Participants & 72 & $\begin{array}{l}\text { M: } 55 \text { in R; } 56 \\
\text { in } S\end{array}$ & $0: 72$ & $\begin{array}{l}\text { Breast cancer } \\
\text { with hot } \\
\text { flashes }\end{array}$ & Not reported & Streitberger & $\begin{array}{l}\text { GV14, } \\
\text { GB20, BL13, } \\
\text { PC7, HT6, } \\
\text { KI7, ST36, } \\
\text { SP6, ear } \\
\text { ShenMen \& } \\
\text { ear } \\
\text { sympathetic } \\
\text { point }\end{array}$ & $18 / 42$ & $18 /$ \\
\hline \multirow[t]{4}{*}{$\begin{array}{l}\text { Dos Santos } \\
\text { Maciel } \\
2016^{47}\end{array}$} & \multirow[t]{4}{*}{$\begin{array}{l}\text { Participants \& } \\
\text { outcome } \\
\text { assessor }\end{array}$} & \multirow[t]{4}{*}{321} & $\begin{array}{l}\overline{\mathrm{x}}: 24.3 \pm 8.8 \\
\text { in } \mathrm{R} ; 25.3 \pm \\
5.4 \text { in } \mathrm{S}\end{array}$ & $\begin{array}{l}5: 18 \text { vs } \\
11: 12\end{array}$ & \multirow[t]{4}{*}{ Healthy } & \multirow[t]{4}{*}{ Naive } & Park & ST25 & $16 / 23$ & $3 / 2$ \\
\hline & & & $\begin{array}{l}\overline{\mathrm{x}}: 24.3 \pm 7.1 \\
\text { in } \mathrm{R} ; 25.1 \pm \\
7.7 \text { in } \mathrm{S}\end{array}$ & $\begin{array}{l}4: 18 \text { vs } \\
3: 20\end{array}$ & & & Park & BL52 & $19 / 22$ & $32 i$ \\
\hline & & & $\begin{array}{l}\overline{\mathrm{x}}: 24.3 \pm 8.8 \\
\text { in } \mathrm{R} ; 26.6 \pm \\
7.8 \text { in } \mathrm{S}\end{array}$ & $\begin{array}{l}5: 18 \text { vs } \\
11: 12\end{array}$ & & & Foam & ST25 & $16 / 23$ & $4 / 2$ \\
\hline & & & $\begin{array}{l}\overline{\mathrm{x}}: 24.3 \pm 7.1 \\
\text { in R; } 29.2 \pm \\
8.0 \text { in } \mathrm{S}\end{array}$ & $\begin{array}{l}4: 18 \text { vs } \\
7: 16\end{array}$ & & & Foam & BL52 & $19 / 22$ & $7 / 2$ \\
\hline $\begin{array}{l}\text { Enblom } \\
2008^{48}\end{array}$ & Participants & 80 & $\begin{array}{l}\overline{\mathrm{x}}: 41 \pm 12.5 \\
(15-67)\end{array}$ & $33: 47$ & Not reported & Naive & Streitberger & PC6 & $13 / 40$ & $13 /$ \\
\hline \multirow{2}{*}{$\begin{array}{l}\text { Enblom } \\
2011^{49}\end{array}$} & \multirow[t]{2}{*}{ Participants } & \multirow[t]{2}{*}{215} & \multirow{2}{*}{$\begin{array}{l}\overline{\mathrm{x}}: 63.7 \pm 13.8 \\
(22-91)\end{array}$} & \multirow[t]{2}{*}{$35: 180$} & \multirow[t]{2}{*}{ Cancer } & Naive & \multirow[t]{2}{*}{ Park } & \multirow[t]{2}{*}{ PC6 } & $50 / 63$ & $8 / 6$ \\
\hline & & & & & & Experienced & & & $23 / 31$ & $2 / 3$ \\
\hline $\begin{array}{l}\text { Fink } \\
2001^{50}\end{array}$ & $\begin{array}{l}\text { Participants \& } \\
\text { statistician }\end{array}$ & 68 & $\overline{\mathrm{x}}: 48.1 \pm 14.1$ & $31: 38^{a}$ & $\begin{array}{l}\text { Episodic/ } \\
\text { chronic } \\
\text { tension-type } \\
\text { headache }\end{array}$ & Not reported & Foam & $\begin{array}{l}\text { GB20, LI4, } \\
\text { LR3 \& TE5 }\end{array}$ & $32 / 32$ & $4 / 3$ \\
\hline $\begin{array}{l}\text { Goddard } \\
2005^{51}\end{array}$ & $\begin{array}{l}\text { Participants \& } \\
\text { outcome } \\
\text { assessor }\end{array}$ & 49 & $(20-60)$ & $29: 20$ & Healthy & Naive & Foam & LI4 & $19 / 24$ & $3 / 2$ \\
\hline $\operatorname{Kim} 2015^{52}$ & $\begin{array}{l}\text { Participants \& } \\
\text { acupuncturist }\end{array}$ & 63 & $\begin{array}{l}\text { M: } 48.9(23- \\
77)\end{array}$ & $27: 35^{b}$ & Healthy & Mixed & Kim & LI4 & $35 / 67^{\wedge}$ & $27 /$ \\
\hline \multirow{2}{*}{$\begin{array}{l}\text { Kreiner } \\
2010^{10}\end{array}$} & \multirow{2}{*}{$\begin{array}{l}\text { Participants \& } \\
\text { acupuncturist }\end{array}$} & \multirow[t]{2}{*}{32} & \multirow[t]{2}{*}{$\overline{\mathrm{x}}: 34(20-62)$} & \multirow[t]{2}{*}{$15: 17$} & \multirow[t]{2}{*}{ Healthy } & \multirow[t]{2}{*}{ Naive } & \multirow[t]{2}{*}{ Foam } & LI4 & $27 / 32^{\wedge}$ & $24 /$ \\
\hline & & & & & & & & ST6 & $27 / 32^{\wedge}$ & $26 /$ \\
\hline Lao $1999^{53}$ & $\begin{array}{l}\text { Participants \& } \\
\text { outcome } \\
\text { assessor }\end{array}$ & 39 & $\begin{array}{l}\overline{\mathrm{x}}: 23.4 \pm 4.7 \\
\text { in } \mathrm{R} ; 24 \pm 3.8 \\
\text { in } \mathrm{S}\end{array}$ & $22: 17$ & $\begin{array}{l}\text { Tooth } \\
\text { extraction }\end{array}$ & Mixed & $\begin{array}{l}\text { Not } \\
\text { classifiable }\end{array}$ & $\begin{array}{l}\text { LI4, ST6, } \\
\text { ST7 \& TE17 }\end{array}$ & $11 / 19$ & $4 / 2$ \\
\hline \multirow[t]{3}{*}{ Lee $2010^{54}$} & \multirow[t]{3}{*}{ Participants } & \multirow[t]{3}{*}{79} & \multirow{3}{*}{$\begin{array}{l}\overline{\mathrm{X}}: 23.7 \pm 4.3 \\
\text { in } \mathrm{R} ; 22.8 \pm \\
4.6 \text { in } \mathrm{S}\end{array}$} & \multirow[t]{3}{*}{$47: 32$} & Not reported & Mixed & Park & LI4 & $25 / 79$ & $18 /$ \\
\hline & & & & & & & & CV12 & $9 / 79$ & $17 /$ \\
\hline & & & & & & & & ST36 & $16 / 79$ & $22 /$ \\
\hline $\begin{array}{l}\text { Liang } \\
2013^{55}\end{array}$ & $\begin{array}{l}\text { Participants \& } \\
\text { statistician }\end{array}$ & 60 & $\overline{\mathrm{x}}: 23.03$ & $12: 48$ & Healthy & Experienced & Park & BL23 & $44 / 60^{\wedge}$ & $12 /$ \\
\hline Liu $2014^{56}$ & $\begin{array}{l}\text { Participants, } \\
\text { outcome }\end{array}$ & 60 & $\begin{array}{l}\overline{\mathrm{x}}: 19.18 \pm \\
15.55(24-\end{array}$ & $30: 30$ & Healthy & Mixed & Foam & LI4 & $59 / 60^{\wedge}$ & $0 / 6$ \\
\hline & $\begin{array}{l}\text { assessor \& } \\
\text { statistician }\end{array}$ & & 74) & & & & & CV12 & $58 / 60^{\wedge}$ & $6 / 6$ \\
\hline & & & & & & & & BL25 & $57 / 60^{\wedge}$ & $7 / 6$ \\
\hline
\end{tabular}

X̃: mean; M: median; (): range; R: real group; S: sham group; a: reported gender ratio did not tally with the sample size; b: baseline characteristic for gender wa: missing (no response); c: drop-out gender was not reported; $\wedge$ : based on the total responses from each participant 


\begin{tabular}{|c|c|c|c|c|c|c|c|c|c|c|}
\hline \multirow[t]{2}{*}{ Study ID } & \multicolumn{2}{|l|}{ Study Design } & \multicolumn{3}{|l|}{ Participants } & \multicolumn{3}{|c|}{ Needling } & \multicolumn{2}{|c|}{$\begin{array}{l}\text { Awareness of thi } \\
\text { of Acupuncture }\end{array}$} \\
\hline & Blinding & $\begin{array}{l}\text { Sample } \\
\text { Size }\end{array}$ & $\begin{array}{l}\text { Mean/Median } \\
\text { Age (Range) } \\
\text { in Years }\end{array}$ & $\begin{array}{l}\text { Gender } \\
(\mathrm{M}: \mathrm{F})\end{array}$ & Health Status & $\begin{array}{l}\text { Acupuncture } \\
\text { Experience }\end{array}$ & $\begin{array}{l}\text { Types of } \\
\text { Sham } \\
\text { Device }\end{array}$ & Location & Real & She \\
\hline & & & & & & & & BL36 & $57 / 60^{\wedge}$ & $3 / 6$ \\
\hline Park $2002^{8}$ & $\begin{array}{l}\text { Participants } \\
\text { and outcome } \\
\text { assessor }\end{array}$ & 58 & $(38-87)$ & $30: 28$ & Acute stroke & Naive & Park & LI4 & $11 / 29$ & $0 / 2$ \\
\hline $\begin{array}{l}\text { Smith } \\
2006^{57}\end{array}$ & Participants & 228 & $\begin{array}{l}\bar{x}: 35.9 \pm 4.7 \\
\text { in } R ; 36.1 \pm \\
4.8 \text { in } S\end{array}$ & $0: 228$ & Infertility & Mixed & Streitberger & $\begin{array}{l}\text { Based on } \\
\text { TCM } \\
\text { diagnosis }\end{array}$ & $40 / 202^{\wedge}$ & $20 /$ \\
\hline $\begin{array}{l}\text { Smith } \\
2011^{58}\end{array}$ & Participants & 92 & $\begin{array}{l}\overline{\mathrm{X}}: 19.5 \pm 2.9 \\
\text { in } \mathrm{R} ; 18.9 \pm \\
3.2 \text { in } \mathrm{S}\end{array}$ & $0: 92$ & $\begin{array}{l}\text { Primary } \\
\text { dysmenorrhoea }\end{array}$ & Mixed & Streitberger & $\begin{array}{l}\text { SP4, ST29, } \\
\text { CV3, BL32, } \\
\text { SP8, SP6 \& } \\
\text { others } \\
\text { based on } \\
\text { the } \\
\text { diagnosis }\end{array}$ & $17 / 41$ & $16 /$ \\
\hline $\begin{array}{l}\text { Streitberger } \\
1998^{7}\end{array}$ & Participants & 60 & $\overline{\mathrm{x}}: 27.55$ & $31: 29$ & Healthy & Naive & Streitberger & LI4 & $54 / 60^{\wedge}$ & $13 /$ \\
\hline $\begin{array}{l}\text { Takakura } \\
2007^{9}\end{array}$ & $\begin{array}{l}\text { Participants \& } \\
\text { acupuncturists }\end{array}$ & 60 & $\overline{\mathrm{x}}: 29.7 \pm 7.5$ & $35: 25$ & Healthy & Experienced & Takakura & TE5 & $48 / 60^{\wedge}$ & $35 /$ \\
\hline $\begin{array}{l}\text { Takakura } \\
2008^{59}\end{array}$ & $\begin{array}{l}\text { Participants \& } \\
\text { acupuncturist }\end{array}$ & 114 & $\overline{\mathrm{x}}: 30.3 \pm 7.9$ & $73: 41$ & Healthy & Experienced & Takakura & TE5 & $78 / 114^{\wedge}$ & $50 /$ \\
\hline \multirow{2}{*}{$\begin{array}{l}\text { Takakura } \\
2011^{60}\end{array}$} & \multirow{2}{*}{$\begin{array}{l}\text { Participants, } \\
\text { acupuncturist, } \\
\text { outcome } \\
\text { assessor }\end{array}$} & \multirow[t]{2}{*}{80} & \multirow[t]{2}{*}{$\overline{\mathrm{x}}: 27.1 \pm 6.9$} & \multirow[t]{2}{*}{$48: 32$} & \multirow[t]{2}{*}{ Healthy } & \multirow[t]{2}{*}{ Experienced } & Takakura & \multirow{2}{*}{$\begin{array}{l}\text { TE meridian } \\
\text { on the } \\
\text { posterior } \\
\text { forearm }\end{array}$} & \multirow[t]{2}{*}{$65 / 80^{\wedge}$} & $38 /$ \\
\hline & & & & & & & $\begin{array}{l}\text { No-touch } \\
\text { Takakura }\end{array}$ & & & $57 /$ \\
\hline $\begin{array}{l}\text { Takakura } \\
2013^{61}\end{array}$ & $\begin{array}{l}\text { Participants \& } \\
\text { acupuncturist }\end{array}$ & 109 & $\overline{\mathrm{x}}: 28.6 \pm 7.5$ & $64: 45$ & Healthy & Experienced & Takakura & TE5 & $85 / 109^{\wedge}$ & $65 /$ \\
\hline \multirow{2}{*}{$\begin{array}{l}\text { Takayama } \\
2014^{62}\end{array}$} & \multirow{2}{*}{$\begin{array}{l}\text { Participants, } \\
\text { acupuncturist } \\
\text { \& assistants }\end{array}$} & \multirow[t]{2}{*}{120} & \multirow[t]{2}{*}{$\overline{\mathrm{x}}: 29.7 \pm 9.3$} & \multirow[t]{2}{*}{$60: 60$} & \multirow{2}{*}{$\begin{array}{l}\text { Functional } \\
\text { neck/shoulder } \\
\text { stiffness }\end{array}$} & \multirow[t]{2}{*}{ Experienced } & Takakura & \multirow{2}{*}{$\begin{array}{l}\text { SI14, SI15, } \\
\text { GB21 \& } \\
\text { BL42 }\end{array}$} & \multirow[t]{2}{*}{$27 / 40$} & $22 /$ \\
\hline & & & & & & & $\begin{array}{l}\text { No-touch } \\
\text { Takakura }\end{array}$ & & & $26 /$ \\
\hline Tan $2009^{63}$ & Participants & 20 & $\begin{array}{l}\text { M: } 22(18- \\
48)\end{array}$ & $6: 14$ & Healthy & Naive & Park & $\begin{array}{l}\text { PC meridian } \\
\text { on the } \\
\text { anterior } \\
\text { forearm }\end{array}$ & $52 / 80^{\wedge}$ & $44 /$ \\
\hline \multirow[t]{2}{*}{$\operatorname{Tan} 2011^{64}$} & \multirow[t]{2}{*}{ Participants } & \multirow[t]{2}{*}{20} & \multirow[t]{2}{*}{$\begin{array}{l}\text { M: } 24(21- \\
28)\end{array}$} & \multirow[t]{2}{*}{$7: 13$} & \multirow[t]{2}{*}{ Healthy } & \multirow[t]{2}{*}{ Naive } & \multirow[t]{2}{*}{ Park } & $\begin{array}{l}\text { TE11, TE12, } \\
\text { TE13 \& } \\
\text { TE14 }\end{array}$ & $24 / 40^{\wedge}$ & $26 /$ \\
\hline & & & & & & & & $\begin{array}{l}\text { BL37, BL55, } \\
\text { BL56 \& } \\
\text { BL57 }\end{array}$ & $25 / 40^{\wedge}$ & $15 /$ \\
\hline Tan $2019^{65}$ & Participants & 40 & $\begin{array}{l}\text { M: } 23(21- \\
40)\end{array}$ & $13: 27$ & Healthy & Not reported & Park & $\begin{array}{l}\text { ST32 to } \\
\text { ST39 }\end{array}$ & $108 / 160^{\wedge}$ & 121 \\
\hline \multirow[t]{3}{*}{ To $2015^{66}$} & Participants \& & 5 & $(23-54)$ & Not & Healthy & Naive & Park & LI4, LI10, & $7 / 16^{\wedge}$ & $8 / 1$ \\
\hline & & 11 & & & Healthy & Experienced & & LI15 \& TE14 & $11 / 31^{\wedge}$ & $17 /$ \\
\hline & & 19 & $(22-74)$ & & $\begin{array}{l}\text { Shoulder } \\
\text { impingement } \\
\text { syndrome }\end{array}$ & Not reported & & $\begin{array}{l}\text { Above } \\
\text { points }+ \\
\text { LV3, SI3, } \\
\text { GB21, Si12 } \\
\text { \& ST38 }\end{array}$ & $8 / 8$ & $0 / 6$ \\
\hline $\begin{array}{l}\text { Tough } \\
2009^{67}\end{array}$ & Participants & 41 & Not reported & $\begin{array}{l}\text { Not } \\
\text { reported }\end{array}$ & Whiplash injury & Mixed & $\begin{array}{l}\text { Not } \\
\text { classifiable }\end{array}$ & $\begin{array}{l}\text { According } \\
\text { to tender } \\
\text { muscle } \\
\text { points }\end{array}$ & $10 / 19$ & $1 / 1$ \\
\hline $\begin{array}{l}\text { Tsukayama } \\
2006^{68}\end{array}$ & Participants & 21 & $\begin{array}{l}\text { M: } 26 \text { (19- } \\
68)\end{array}$ & $15: 6$ & Healthy & Experienced & Park & LI4 & $21 / 21^{\wedge}$ & $12 /$ \\
\hline & & 20 & $\begin{array}{l}\text { M: } 24 \text { (19- } \\
37)\end{array}$ & $15: 5$ & & & & BL23 & $14 / 20^{\wedge}$ & $8 / 2$ \\
\hline
\end{tabular}
missing (no response); c: drop-out gender was not reported; ${ }^{\wedge}$ : based on the total responses from each participant 


\begin{tabular}{|c|c|c|c|c|c|c|c|c|c|c|}
\hline \multirow[t]{2}{*}{ Study ID } & \multicolumn{2}{|l|}{ Study Design } & \multicolumn{4}{|l|}{ Participants } & \multicolumn{2}{|l|}{ Needling } & \multicolumn{2}{|c|}{$\begin{array}{l}\text { Awareness of th } \\
\text { of Acupuncture }\end{array}$} \\
\hline & Blinding & $\begin{array}{l}\text { Sample } \\
\text { Size }\end{array}$ & $\begin{array}{l}\text { Mean/Median } \\
\text { Age (Range) } \\
\text { in Years }\end{array}$ & $\begin{array}{l}\text { Gender } \\
(\mathrm{M}: \mathrm{F})\end{array}$ & Health Status & $\begin{array}{l}\text { Acupuncture } \\
\text { Experience }\end{array}$ & $\begin{array}{l}\text { Types of } \\
\text { Sham } \\
\text { Device }\end{array}$ & Location & Real & She \\
\hline $\begin{array}{l}\text { Vase } \\
2015^{69}\end{array}$ & $\begin{array}{l}\text { Participants \& } \\
\text { acupuncturists }\end{array}$ & 67 & $\overline{\mathrm{x}}: 25.8 \pm 5.0$ & $31: 36$ & $\begin{array}{l}\text { Pain after } \\
\text { mandibular } \\
\text { third molar } \\
\text { removal }\end{array}$ & Naive & Takakura & $\begin{array}{l}\text { ST44, LI4, } \\
\text { ST7, ST6 \& } \\
\text { TE17 }\end{array}$ & $22 / 32$ & $21 /$ \\
\hline $\begin{array}{l}\text { White } \\
1996^{70}\end{array}$ & $\begin{array}{l}\text { Participants \& } \\
\text { outcome } \\
\text { assessor }\end{array}$ & 10 & $\begin{array}{l}\overline{\mathrm{x}}: 57.2 \pm 13.6 \\
\text { in } \mathrm{R} ; 5.4 \pm \\
19.9 \text { in } \mathrm{S}\end{array}$ & $2: 7^{c}$ & $\begin{array}{l}\text { Episodic } \\
\text { tension-type } \\
\text { headache }\end{array}$ & Naive & $\begin{array}{l}\text { Cocktail } \\
\text { stick }\end{array}$ & $\begin{array}{l}\text { LI4 with } \\
\text { local points } \\
\text { like GB14, } \\
\text { GB20, } \\
\text { GB21, EX- } \\
\text { HN3 } \\
\text { YinTang \& } \\
\text { EX-HN5 } \\
\text { TaiYang }\end{array}$ & $4 / 4$ & $1 / 5$ \\
\hline $\begin{array}{l}\text { White } \\
2003^{71}\end{array}$ & Participants & 37 & $\begin{array}{l}\overline{\mathrm{x}}: 65.8 \pm 8.3 \& \\
64.4 \pm 12.7 \\
(37-79)\end{array}$ & $14: 23$ & $\begin{array}{l}\text { Chronic/stable } \\
\text { hip/knee joint } \\
\text { pain }\end{array}$ & Mixed & Streitberger & $\begin{array}{l}\text { GB30, } \\
\text { GB31, } \\
\text { GB34, BL34, } \\
\text { BL39, BL40, } \\
\text { BL60, ST31, } \\
\text { ST35, ST36, } \\
\text { ST41, SP9 } \\
\text { \& EX-LE5 } \\
\text { XiYan }\end{array}$ & $25 / 37^{\wedge}$ & $14 /$ \\
\hline \multirow[t]{2}{*}{$\begin{array}{l}\text { White } \\
2007^{72}\end{array}$} & Participants & 20 & $\begin{array}{l}\overline{\mathrm{x}}: 50.8 \pm 14.9 \\
(35-80)\end{array}$ & 9:11 & Healthy & Mixed & Streitberger & LI4 & $16 / 20^{\wedge}$ & $10 /$ \\
\hline & & 14 & $\begin{array}{l}\overline{\mathrm{x}}: 59.4 \pm 5.7 \\
(49-67)\end{array}$ & 3:11 & $\begin{array}{l}\text { Chronic } \\
\text { osteoarthritis }\end{array}$ & Mixed & & & $14 / 14^{\wedge}$ & $3 / 1$ \\
\hline Xie $2013^{73}$ & Participants & 60 & $\begin{array}{l}\overline{\mathrm{x}}: 23.07 \pm \\
0.753 \text { in } \mathrm{R} \\
23.13 \pm 1.056 \\
\text { in S }(22-25)\end{array}$ & $20: 40$ & Healthy & Experienced & Streitberger & BL23 & $45 / 60^{\wedge}$ & $11 /$ \\
\hline
\end{tabular}

x: mean; M: median; (): range; R: real group; S: sham group; a: reported gender ratio did not tally with the sample size; b: baseline characteristic for gender wa: missing (no response); c: drop-out gender was not reported; ${ }^{\wedge}$ : based on the total responses from each participant

All recruited subjects were adults. The mean age ranged from 18.9 to 65.8 years old. In some of the studies, diseased subjects were recruited such as cancer $^{46,49}$, headach $\mathrm{e}^{50,70}$, tooth extraction ${ }^{53,69}$, stroke ${ }^{8}$, infertility ${ }^{57}$, dysmenorrhoea ${ }^{58}$, neck/shoulder stiffness ${ }^{62}$, shoulder impingement syndrome ${ }^{66}$, whiplash injury ${ }^{67}$ and joint pain/osteoarthritis ${ }^{71,72}$; whereas the rest of them were either healthy or not reported. Most studies reported acupuncture experience of the subjects, for example, naive (never experienced acupuncture before), experienced (at least experienced acupuncture once before) or mixed.

A variety of non-penetrating sham acupuncture devices was used in the studies. They were classified and named according to the name of the authors (Park, Streitberger, Takakura and Kim) or materials (foam and cocktail stick). The studies had chosen acupoints or non-acupoints located on the head, abdomen, back, upper limbs, lower limbs and ears. The coding and naming of acupoints were converted if they did not follow a Proposed Standard International Acupuncture Nomenclature ${ }^{74}$ by the World Health Organization (WHO). Some selected acupoints based on syndrome differentiation of Chinese medicine ${ }^{57,58}$ or tender muscle point ${ }^{67}$ and, hence, did not have constant points throughout the study.

The number of participants who guessed correctly the type of acupuncture (real or sham) was listed in Table 2. Some studies were recorded in the form of the total number of responses from the participants due to cross-over study design 7,10,44,45,55,56,68,71-73, using a mixture of real and sham acupuncture devices $9,52,59-61,63-66$ or multiple times of evaluation ${ }^{57}$. These responses were extracted and analysed because the blinding effectiveness of a particular sham device ideally should be maintained even after several times of evaluation. Most studies recorded their results in Yes-No format, whereas the 15 of them $8,44,46,48,49,53,54,57,58,60,62,67,70,71,73$ accepted "not sure" as one of the answers from the participants.

\section{Quality Assessment}

Quality and risk of bias of the eligible studies were assessed independently by two researchers at outcome level by using Cochrane risk of bias tool ${ }^{14}$. Quality assessments included random sequence generation, allocation concealment, blinding of participants and personnel, blinding of outcome assessment, incomplete outcome data, selective reporting and other items. Studies were graded as low, unclear or high risk of bias. Discrepancies were resolved via discussion among the researchers. Publication bias across the studies was presented as funnel plot.

\section{Data Analysis}

Meta-analysis was performed using RevMan 5.4. $1^{15}$. The effectiveness of blinding in sham/placebo acupuncture compared to real acupuncture was estimated with the odds ratios (OR) and its $95 \%$ confidence intervals. OR $>1$ means blinding is more likely to occur in the intervention arm (sham group) than 
in the comparator arm (real group). Trials which the patients had no events in both intervention and comparator arms were excluded from the metaanalysis Heterogeneities were assessed using the chi-squared $\left(\mathrm{X}^{2}\right)$ test and the inconsistency index $\left(\mathrm{I}^{2}\right)$ statistic. A two-tailed $\mathrm{P}$ value of less than 0.05 was considered as statistically significant. Subgroup analyses were performed on the factors which could influence the blinding effectiveness of participants.

\section{Blinding Effectiveness of Sham Acupuncture Devices}

The OR of overall blinding effectiveness of non-penetrating sham acupuncture devices (Fig. 5) was $5.11[3.36,7.76]$ with a P-value (<0.00001), which indicated that the blinding was more likely to occur in the sham group and the result was statistically significant. However, the studies were not homogenous and the heterogeneity was high in the meta-analysis including the subgroup analyses, hence, a random effect model was selected. After switching to the random effect model, the heterogeneity was still high $\left(I^{2}=88 \%\right)$ in the overall blinding effectiveness analysis, so subgroup analyses were performed to evaluate the factors which may influence the blinding effectiveness of sham acupuncture devices. For example, acupuncture experience of the participants, the health status of the participants and the location of acupoints.

A comparison between sham acupuncture devices was performed through evaluating and comparing the individual blinding effectiveness of sham acupuncture devices. Kim and cocktail stick devices were excluded because they only possessed one study each. As shown in Fig. 6 , the foam device was the most successful device in blinding the participants because it has the highest OR (44.78) [10.03, 199.92], followed by Streitberger (4.69) [1.87, 11.78], Park (3.16) $[1.61,6.21]$, Takakura (2.66) $[1.98,3.55]$ and no-touch Takakura $(1.47)[0.82,2.62]$. The P-value was statistically significant $(<0.05)$ in all devices except for the no-touch Takakura device. The heterogeneity was high in Park, Streitberger and foam but low in Takakura and no-touch Takakura. The heterogeneity across the subgroups was high $\left(I^{2}=79.3 \%\right)$.

\section{Blinding Effectiveness in Different Types of Participants}

Naive participants demonstrated superiority in achieving blinding during acupuncture clinical trials with an OR 5.73 [2.76, 11.89] compared to experienced subjects 3.22 [1.94, 5.37] (Fig. 7). Both naive and experienced participants were successful in blinding because they were both statistically significant ( $P<$ 0.00001). The heterogeneity was high in both groups individually but did not differ much between the two subgroups $\left(I^{2}=37.7 \%\right)$.

As for the health status of participants, both healthy and diseased participants were statistically significantly successful in blinding $(P<0.00001)$ using sham acupuncture devices (Fig. 8). Healthy participants were blinded slightly better than diseased participants with an OR 6.28 [3.62, 10.91] compared to 5.79 [2.71, 12.35]. The heterogeneity was high within the subgroups but low when comparing the two subgroups $\left(I^{2}=0 \%\right)$.

\section{Blinding Effectiveness in Different Locations of Acupoints}

The acupoints of the included studies had been classified according to its regions, for example, head and neck, chest, abdomen, back, upper limbs and lower limbs. Studies which utilised acupoints from multiple parts of the body were excluded. Besides, there was only one study which had used acupoints purely from the head and neck region, therefore it was excluded from the meta-analysis. Among the remaining regions, the abdomen demonstrated a better blinding effect with an OR 11.33 [0.71, 180.77], followed by the back $(9.10)[3.14,26.36]$, the upper limbs $(4.31)[2.50,7.43]$ and the lower limbs (3.69) [0.82, 16.62] (Fig. 9). The back and the upper limbs exhibited a statistically significant result $(P<0.0001)$, whereas the results of the abdomen and the lower limbs were not statistically significantly different $(P=0.09)$. However, heterogeneity was high in all of the individual subgroups but low across the subgroups $\left(I^{2}=0 \%\right)$.

\section{Discussion}

\section{Blinding Effectiveness of Sham Acupuncture Devices}

\section{Overall Blinding Effectiveness of Sham Acupuncture Devices}

When conducting acupuncture clinical trials, subjects from both real acupuncture group and sham acupuncture group should believe that they receive real acupuncture to mimic the actual scenario which takes place during acupuncture treatment. As shown in Fig. 5, the existing non-penetrating sham acupuncture devices such as Park, Streitberger, Takakura, foam and cocktail stick displayed a result which favoured the sham group and was also statistically significant. In other words, more participants in the sham acupuncture group who did not identify the sham acupuncture treatment correctly compared to the real group and hence, they were blinded. As a result, non-penetrating sham acupuncture devices can act as an effective placebo control method to be applied in acupuncture clinical trials, especially in replacing other less effective types of control methods such as using no treatment, standard/conventional treatment or skin-penetrating sham acupuncture as the control.

\section{Comparison between Sham Acupuncture Devices}

Among all the non-penetrating sham acupuncture devices included in the data analysis, the foam device demonstrated the best ability in achieving the blinding of participants. There were five studies ${ }^{10,47,50,51,56}$ which used the foam device in their control group. The foam device is usually self-prepared by the researchers, so there are some variations in design. It is made out of a certain thickness of foam with double-sided adhesive tape at the bottom (Fig. 10). The foam pad can act as a supportive material to hold the needle in place even in the placebo group, whereas the adhesive tape can stick the device on the skin. The real foam device utilises a real acupuncture needle with a sharp tip which can penetrate the skin of participants; the placebo foam device uses a shorter blunted acupuncture needle which cannot penetrate the skin. Ultimately, the appearance after needle insertion will remain the same and hence, achieve blinding of participants in terms of vision. After needle insertion, the real device will penetrate the skin with a certain depth, whereas the placebo device will only touch the skin to blind the participants by mimicking the feeling of penetration. In the studies of Fink ${ }^{50}$ and Goddard ${ }^{51}$, the placebo needles were gently twisted to enhance the feeling of penetration. Besides having a good blinding effect, the foam device is also less pricey and easily accessible compared to the 
other devices, so it can be a good option to be used in the control group of acupuncture clinical trials. However, owing to the preparation of the foam device is usually self-made, hygiene in preparing the device will become the main concern. All the equipment must be sterilised adequately before being applied to the participants.

The other sham acupuncture devices such as Streitberger, Park and Takakura (Fig. 11) are also effective in blinding the participants. Their sham devices look identical with the real devices. Similar to the foam device, their sham devices possess shorter blunted-tip needles which touch on the skin to mimic penetrating sensation. Hence, all of them can be applied in clinical trials. The characteristics of the foam, Streitberger, Park and Takakura devices have been summarised in Table 3. On the other hand, the sham device of no-touch Takakura was not statistically significantly superior to the real device $(P=0.19)$, so it is not recommended to be used in clinical trials. No-touch sham Takakura device has no contact with the skin of participants. It may be useful in achieving visual blinding but not tactile blinding.

Table 3

Analysis of Sham Acupuncture Devices

\begin{tabular}{|c|c|c|c|c|}
\hline Devices & Characteristics & Price ${ }^{75}$ & Advantages & Limitations \\
\hline Foam & $\begin{array}{l}\text { Needle is supported by an opaque guide tube which is attached to the } \\
\text { foam base; adhesive tape at the bottom }\end{array}$ & N/A & $\begin{array}{l}\text { Inexpensive, easily } \\
\text { accessible and self- } \\
\text { prepared }\end{array}$ & $\begin{array}{l}\text { Potential risk of hygienic issue } \\
\text { and inconsistency of quality of } \\
\text { the foam }\end{array}$ \\
\hline Streitberger & $\begin{array}{l}\text { Sham: retractable needle supported by plastic ring covered with } \\
\text { plastic sheet as the base; adhesive tape at the bottom }\end{array}$ & $\begin{array}{l}\$ 6.3 \\
\text { per } \\
\text { needle }\end{array}$ & $\begin{array}{l}\text { Can insert in } \\
\text { different angles }\end{array}$ & $\begin{array}{l}\text { Sham needle attachment is not } \\
\text { firm }\end{array}$ \\
\hline Park & $\begin{array}{l}\text { Needle is supported by an opaque guide tube and a Park tube which is } \\
\text { connected with the ring base; adhesive tape at the bottom }\end{array}$ & $\begin{array}{l}\$ 2.9 \\
\text { per } \\
\text { needle }\end{array}$ & $\begin{array}{l}\text { Able to perform } \\
\text { simple needle } \\
\text { manipulations }\end{array}$ & Can only insert perpendicularly \\
\hline Takakura & $\begin{array}{l}\text { Needle is supported by an opaque guide tube which is filled up with } \\
\text { stuffings at upper and lower ends and the tube is connected to an } \\
\text { adhesive pedestal }\end{array}$ & N/A & $\begin{array}{l}\text { Potentially blind the } \\
\text { acupuncturist }\end{array}$ & Can only insert perpendicularly \\
\hline \multicolumn{5}{|c|}{ N/A: not available } \\
\hline
\end{tabular}

In short, each sham acupuncture design has its own advantages and limitations. Researchers should take that into consideration when designing the experimental and control methods. Due to high heterogeneity of the studies across the subgroups (Fig. 6), the OR value may be influenced by other factors and hence, the results of blinding effectiveness of sham devices can only serve as a reference.

\section{Limitations of the Existing Sham Acupuncture Devices}

The limitations of the existing sham acupuncture devices can be discussed in several aspects, including the suitability in performing electro-acupuncture, needling location, needling angle and blinding effect in acupuncturists.

The sham devices of foam, Streitberger, Park and Takakura solely rely on the adhesive double-sided tape or pedestal to attach to the skin. The attachment is not as firmly as those in the real acupuncture, therefore, sham electro-acupuncture is difficult to perform by using these devices. Moreover, the adhesive tape may not be suitable for hairy skin or areas which are not flat. As a result, using sham acupuncture devices on the scalp, hairy regions and the skin with a great curvature such as the ears, fingers and toes can be challenging.

Apart from that, needling angle is usually limited to perpendicular for the foam, Park and Takakura devices due to the presence of guide tube, so only acupoints that allow perpendicular insertion can be selected when using these devices. Acupoints such as LU7 LieQue and EX-HN3 YinTang or acupoints on the scalp which require oblique or transverse insertion cannot be chosen in the trials. On the contrary, Streitberger device, which does not have a guide tube, can perform perpendicular and oblique insertions. Yet, having no guide tube can also make the needle unstable especially in the sham group, increasing the risk of exposure of grouping allocation.

Last but not least, most of the sham acupuncture devices did not demonstrate the ability to blind acupuncturists, except for the Takakura device. The Takakura device has added a lower stuffing within its guide tube to mimic the feeling of skin penetration when the acupuncturist pushes the sham needle into the lower stuffing. In other words, the performance bias will be high in the acupuncture clinical trials that use other types of sham acupuncture devices.

\section{Considerations of Designing Sham Acupuncture Controlled Clinical Trials}

\section{Guidelines of Sham Acupuncture Controlled Clinical Trials}

In 1995, the Guidelines for Clinical Research on Acupuncture ${ }^{4}$ by the WHO stated that placebo acupuncture should fulfil two criteria: it must be a less effective form of acupuncture and also mimic acupuncture in a credible manner. Zhang's paper ${ }^{76}$ also stated that placebo acupuncture should have no or minimal specific treatment effects on the tested disease and the treatment and control groups should be identical to achieve blinding. To evaluate the true efficacy of acupuncture, the difference in specific effects shall be maximised but the difference in non-specific effects shall be minimised (Fig. 12).

Page $9 / 20$ 
Determining a research question is important before selecting the type of control because each control method can be used to answer different types of research questions. As shown in Table 4, no treatment and standard treatment as control can rule out regression to the mean and study the general effectiveness of acupuncture; non-penetrating sham as control can rule out regression to the mean and psychological responses (placebo effect) and study the efficacy of acupuncture, including skin-penetrating physiological effects and acupoint specific effects; lastly, penetrating sham as control can rule out three other aspects and study specifically on the efficacy of acupoint specific effects ${ }^{77,78}$. As far as the authors know, non-penetrating sham acupuncture is the only method that can eliminate the placebo effect and meanwhile, minimise the physiological responses being generated. So, it can be widely used in a broad range of acupuncture clinical trials that study the specific effects of acupuncture. On the other hand, penetrating sham acupuncture (e.g. shallow needling/minimal acupuncture and needling on non-acupoints) is suitable to study narrower specific effects of acupuncture that will not be generated by skin penetration. Do note that the acupuncturists of penetrating sham acupuncture are not blinded most of the time owing to different needling techniques and locations in the sham group and hence, it may lead to performance bias of personnel. In addition, it has to be ensured that skin penetration will not trigger any desired specific effects of the study, if not it will result in no significant difference in both arms.

Table 4

Study Focus of Different Types of Acupuncture Control

\begin{tabular}{|lllll|}
\hline Types of Control & \multicolumn{2}{l}{ Aspects } & & \\
\cline { 2 - 5 } & Regression to the Mean & Psychological Responses & Physiological Responses* & Acupoint Specific Effects \\
\hline No treatment & $X$ & Effectiveness & Efficacy & Efficacy \\
\hline Standard treatment & $X$ & Effectiveness & Efficacy & Efficacy \\
\hline Non-penetrating sham & $X$ & $X$ & Efficacy & Efficacy \\
\hline Penetrating sham & $X$ & $X$ & $X$ & \\
\hline *, physiological responses caused by the skin penetration & & \\
\hline Note: the boxes with "X" in Table 4 indicate the areas that are eliminated when comparing with the real acupuncture group.
\end{tabular}

\section{Factors which May Influence the Blinding Effectiveness of Sham Acupuncture Devices}

Besides the selection of sham acupuncture method and device, there are other factors which may also play a role in achieving successful blinding, for example, acupuncture experience and health status of the participants and needling location. As shown in Fig. 7, the naive participants are more easily blinded than the experienced participants. The experienced participants are more familiar with the acupuncture process and DeQi (needling) sensation and hence, they are more likely to guess the grouping accurately. However, both groups showed a significant difference in blinding effectiveness. Ideally, naive subjects should be recruited in acupuncture clinical trials, but experienced participants can also be considered if naive ones are not available or sufficient.

Next, both healthy and diseased participants demonstrated a significant difference in blinding effectiveness, in which the healthy ones were slightly superior to the other group (Fig. 8). Healthy subjects should be prioritised when designing acupuncture clinical trials, nonetheless, recruiting diseased participants is unavoidable when studying the efficacy of acupuncture on a particular disease.

Lastly, the locations of acupoints may also influence the effectiveness of blinding. As shown in Fig. 9, the OR values of the abdomen (11.33) [0.71, 180.77] and the back $(9.1)[3.14,26.36]$ were higher compared to the upper limbs $(4.31)[2.50,7.43]$ and the lower limbs $(3.69)[0.82,16.62]$. This phenomenon is associated with the higher sensitivity of the skin and stronger needling sensations in the four limbs due to rich nervous distribution. Yet, only the back and upper limbs showed statistically significant different P-values.

\section{Limitations of the Study}

Four studies had been excluded due to language barrier and seven studies had been excluded due to inaccessibility of the full-text articles. Excluding articles other than English and Chinese may introduce language bias. Excluding inaccessible articles may also reduce the precision of combined estimates of blinding effectiveness. However, the authors were not able to overcome it due to limited resources. The included studies utilised different ways to present data, so some data had to be converted before performing the meta-analysis. Also, some studies presented their results in terms of the number of participants, whereas some were based on the total responses from the participants.

A variety of study designs also led to high heterogeneity in the results of the meta-analysis. Other potential influencing factors which might contribute to the heterogeneity such as the diameter of acupuncture needle, the depth of needle insertion, the duration of needle retention, needle manipulation techniques and the number of treatments were not analysed in the study. For instance, the participants may be aware of the grouping by observing the procedure and treatment effects after having multiple and long-time treatment.

The number of articles in some of the subgroups was small. For example, Kim device and cocktail stick device in the analysis of the types of sham acupuncture devices possessed solely one study; besides, the head and neck, abdomen and lower limbs in the analysis of the locations of acupoints possessed a small number of studies.

\section{Conclusions}


Non-penetrating sham acupuncture devices are valid placebo control for acupuncture clinical trials. It should be applied in future clinical trials because it can blind the participants and meanwhile, produces lesser physiological responses. The foam device has a better blinding effect, followed by the Streitberger, Park and Takakura devices. Sham needles with no skin contact could not successfully blind the participants. Naive, experienced, healthy and diseased participants all can be used in acupuncture clinical trials but naive and healthy participants can be blinded more easily. Choosing acupoints from the back can blind participants more easily compared to the other areas.

Future clinical trials can study and compare the blinding effectiveness of sham acupuncture devices by performing multiple sham groups with a larger sample size to evaluate all different types of sham devices under the same setting. This can eliminate heterogeneity and show real blinding efficacy of these devices. Besides, researchers can also try to develop a better type of non-penetrating sham acupuncture device with the considerations of the blinding efficacy, strengths and limitations as mentioned in the results and discussion.

\section{Abbreviations}

PRISMA: Preferred Reporting Items for Systematic Reviews and Meta-Analyses; RCT: Randomised Controlled Trial; OR: Odd Ratio; WHO: World Health Organization

\section{Declarations}

\section{Acknowledgements}

The authors would like to thank the authors of included and excluded studies who had shared their research findings.

\section{Authors' Contributions}

GYH provided the idea of the study. SNA provided the study design. GYH conducted the literature search from the databases. GYH, SNA and SYS performed article screening, data extraction and quality assessment. GYH and SNA performed meta-analysis. GYH wrote the manuscript. All authors approved the final submission.

\section{Funding}

The study was funded by the Institute for Research, Development and Innovation of International Medical University, Malaysia.

\section{Availability of data and materials}

All data generated or analysed during this study are included in this published article.

\section{Ethics approval and consent to participate}

Not applicable.

\section{Consent for publication}

All authors agree for publication in BMC Complementary Medicine and Therapies.

\section{Competing interests}

All authors declare that there are no conflicts of interest.

\section{Author details}

1Chinese Medicine Department, Centre for Complementary and Alternative Medicine, International Medical University, Kuala Lumpur, Malaysia. 2Division of Pathology, School of Medicine, International Medical University, Kuala Lumpur, Malaysia. 3Chinese Medicine Department, Centre for Complementary and Alternative Medicine, International Medical University, Kuala Lumpur, Malaysia.

\section{References}

1. Zhuang Y, Xing JJ, Li J, Zeng BY, Liang FR. Chapter one - history of acupuncture research. Int Rev Neurobiol. 2013 Nov 8;111:1-23. DOI: 10.1016/B978-012-411545-3.00001-8

2. Hariton E, Locascio JJ. Randomised controlled trials-the gold standard for effectiveness research. BJOG. 2018 Dec 1;125(13):1716. DOI: 10.1111/14710528.15199

3. Karanicolas PJ, Farrokhyar F, Bhandari M. Blinding: who, what, when, why, how? Can J Surg. 2010 0ct;53(5):345-8.

4. Guidelines for clinical research on acupuncture. [ebook]. Western Pacific: World Health Organization; 1995. Available from: https://apps.who.int/iris/bitstream/handle/10665/207013/9290611146_eng.pdf?sequence=1\&isAllowed=y

5. Lund I, Näslund J, Lundeberg T. Minimal acupuncture is not a valid placebo control in randomised controlled trials of acupuncture: a physiologist's perspective. Chin Med. 2009 Jan 30;4:1. DOI: 10.1186/1749-8546-4-1 
6. Lundeberg T, Lund I, Sing A, Näslund J. Is placebo acupuncture what it is intended to be? Evidence-Based Complement Altern Med. 2011 Jun 18;2011:1-5. DOI: 10.1093/ecam/nep049

7. Streitberger K, Kleinhenz J. Introducing a placebo needle into acupuncture research. Lancet. 1998 Aug 1;352(9125):364-5. DOI: 10.1016/S01406736(97)10471-8

8. Park J, White A, Stevinson C, Ernst E, James M. Validating a new non-penetrating sham acupuncture device: two randomised controlled trials. Acupunct Med. 2002 Dec;20(4):168-74. DOI: 10.1136/aim.20.4.168

9. Takakura N, Yajima H. A double-blind placebo needle for acupuncture research. BMC Complement Altern Med. 2007 Oct 10;7:31. DOI: 10.1186/14726882-7-31

10. Kreiner M, Zaffaroni A, Alvarez R, Clark G. Validation of a simplified sham acupuncture technique for its use in clinical research: a randomised, single blind, crossover study. Acupunct Med. 2010 Mar;28(1):33-6. DOI: 10.1136/aim.2009.001735

11. Zhang GS, Zhang CS, Tan HY, Wang Y, DaCosta C, Zhang AL, et al. Systematic review of acupuncture placebo devices with a focus on the credibility of blinding of healthy participants and/or acupuncturists. Acupunct Med. 2018 Aug;36(4):204-14. DOI: 10.1136/acupmed-2017-011484

12. Gong XL, Pan ZH, Shen Y, Wang S. Blinding effect of non-penetrating sham needle in the randomized controlled trials of acupuncture: a systematic review. J Acupunct Tuina Sci. 2014 Feb 1;12(1):8-11. DOI: 10.1007/s11726-014-0738-1

13. Moher D, Liberati A, Tetzlaff J, Altman DG. Preferred reporting items for systematic reviews and meta-analyses: the PRISMA statement. BMJ. 2009 Jul 21;339:b2535. DOl: 10.1136/bmj.b2535

14. Higgins JP, Altman DG, Gøtzsche PC, Jüni P, Moher D, Oxman AD, et al. The Cochrane Collaboration's tool for assessing risk of bias in randomised trials. BMJ. 2011 Oct 18;343:d5928. DOI: 10.1136/bmj.d5928

15. Review Manager (RevMan) [Computer program]. Version 5.4.1, The Cochrane Collaboration, 2020.

16. Chae YB, Kim YJ, Choe IH, Lim S, Lee SJ, Lee HJ, et al. A comparison study of acupuncture sensation scale between real acupuncture and sham needle. Korean J Merid Acupoint. 2006;23(4):85-99.

17. Dilli CR, Childs R, Berk J, Christian MK, Nguyen N, Brown RP, et al. Does prior acupuncture exposure affect perception of blinded real or sham acupuncture? Acupunct Med. 2014 Apr;32(2):155-9. DOI: 10.1136/acupmed-2013-010449

18. Fink M, Rollnik JD, Bijak M, Borstädt C, Däuper J, Guergueltcheva V, et al. Needle acupuncture in chronic poststroke leg spasticity. Arch Phys Med Rehabil. 2004 Apr;85(4):667-72. DOI: 10.1016/j.apmr.2003.06.012

19. Fink M, Karst M. Needling sensations following real and placebo acupuncture - a randomised single-blinded two-period cross-over pilot study. Deutsche Zeitschrift für Akupunktur. 2005;48(2):6-10.

20. Foster NE, Thomas E, Barlas P, Hill JC, Young J, Mason E, et al. Acupuncture as an adjunct to exercise based physiotherapy for osteoarthritis of the knee: randomised controlled trial. BMJ. 2007 Sep 1;335(7617):436. DOI: 10.1136/bmj.39280.509803.BE

21. Francia A, Kreiner M, Fernández I, Massa F. Validation of a new placebo acupuncture method with potential for double blinding. Phase II: practitioner blinding. Odontoestomatología. 2018 Dec;20(32): 42-50.

22. Grillo CM, Zotelli VLR, Lúcia Bressiani Gil M, de Sousa MDLR. Would a placebo acupuncture needle be able to induce Deqi? J Acupunct Meridian Stud. 2018 Oct;11(5):273-9. DOI: 10.1016/j.jams.2018.06.007

23. Hu H, Hu T, Han D, Gao H. Establishment of appropriate sham acupuncture and successful patient blinding. Acupunct Med. 2020 Jun 12:964528420924035. DOI: 10.1177/0964528420924035

24. Jiang W, Chen W, Coeytaux R. Acupuncture credibility scale scores associated with TCM acupuncture versus a sham acupuncture procedure: implication for clinical trial design. J Integrat Med. 2014(3):309.

25. Lee HS, Park J, Shim SY, Kim YJ, Lee JM, Lee H, et al. Non-penetrating sham needle - is it an adequate sham control for acupuncture research in Korean people? Focus Altern Complement Therap. 2010 Jun 14;11(1).

26. Leem J, Park J, Han G, Eun S, Makary MM, Park K, et al. Evaluating validity of various acupuncture device types: a random sequence clinical trial. BMC Complement Altern Med. 2016 Feb 2;16:43. DOI: 10.1186/s12906-016-1026-z

27. Lim JA, Seo JC, Lee EY, Lee HS, Moon HC, Choi SM, et al. The study of the sham acupuncture for double blind. J Acupunct Research. 2006 Oct 20;23(5):127-44.

28. Park J, White A, Lee H, Yamashita H, Ernst E. Credibility of a newly developed sham needle. J Japan Societ Acupunct Moxibust. 2000;50(1):111-4.

29. Park J, White A, Stevinson C, Ernst E, James M. Validating a new sham (placebo) acupuncture device: two randomized controlled trials. Acupunct Med. 2002 Aug 1;20(2-3):123-39. DOI: 10.1136/aim.20.2-3.123

30. Park JH, Chae Y, Park HJ, Lee HJ. A visual factor of blinding of the non-penetrating placebo needle. Korean J Acupunct. 2008;25(4):175-85.

31. Park JJ. Developing and validating a sham acupuncture needle. Acupunct Med. 2009 Sep;27(3):93. D0I: 10.1136/aim.2009.001495.

32. Park J, White AR, James MA, Chambers J, Stedman V, Lee H, et al. Credibility of a newly developed sham-needle unit for controlled clinical trials. Focus on Alternat Complement Therap. 2001 Mar;6(1):88-9.

33. Streitberger K, Friedrich-Rust M, Bardenheuer H, Unnebrink K, Windeler J, Goldschmidt H, et al. Effect of acupuncture compared with placebo-acupuncture at P6 as additional antiemetic prophylaxis in high-dose chemotherapy and autologous peripheral blood stem cell transplantation: a randomized controlled single-blind trial. Clin Cancer Res. 2003 Jul;9(7):2538-44.

34. Streitberger K, Diefenbacher M, Bauer A, Conradi R, Bardenheuer H, Martin E, et al. Acupuncture compared to placebo-acupuncture for postoperative nausea and vomiting prophylaxis: a randomised placebo-controlled patient and observer blind trial. Anaesthesia. 2004 Feb;59(2):142-9. DOI:

Page $12 / 20$ 
10.1111/j.1365-2044.2004.03577.x

35. Takakura N, Takayama M, Kawase A, Kaptchuk TJ, Yajima H. Double blinding with a new placebo needle: a further validation study. Acupunct Med. 2010 Sep;28(3):144-8. DOI: 10.1136/aim.2009.001230

36. Takakura N, Takayama M, Kawase A, Yajima H. Tapping-in method (skin penetration technique) with a placebo needle for double-blind acupuncture trials. J Altern Complement Med. 2013 Apr;19(4):308-12. DOI: 10.1089/acm.2012.0056

37. Takakura N, Takayama M, Kawase A, Kaptchuk TJ, Kong J, Yajima H. Design of a randomised acupuncture trial on functional neck/shoulder stiffness with two placebo controls. BMC Complement Altern Med. 2014 Jul 16;14:246. DOI: 10.1186/1472-6882-14-246

38. Tsukayama H, Yamashita H, Kimura T, Otsuki K. Factors that influence the applicability of sham needle in acupuncture trials: two randomized, singleblind, crossover trials with acupuncture-experienced subjects. Clin J Pain. 2006 May;22(4):346-9. DOI: 10.1097/01.ajp.0000176359.94644.mL

39. Vickers AJ, Feinstein MB, Deng GE, Cassileth BR. Acupuncture for dyspnea in advanced cancer: a randomized, placebo-controlled pilot trial [ISRCTN89462491]. BMC Palliat Care. 2005 Aug 18;4:5. DOI: 10.1186/1472-684X-4-5

40. White AR, Resch KL, Chan JC, Norris CD, Modi SK, Patel JN, et al. Acupuncture for episodic tension-type headache: a multicentre randomized controlled trial. Cephalalgia. 2000 Sep;20(7):632-7. DOI: 10.1111/j.1468-2982.2000.00097.x

41. Wong YM. Evaluating blinding effectiveness of a novel Ryodoraku sham needle device. Acupunct Med. 2018 Feb;36(1):56-7. DOI: 10.1136/acupmed2016-011328

42. Yan L, Sun J, Xu M, Guan SM, Ma LH. Placebo Deqi sensation evoked by a new sham needle: a randomized controlled trial. J Clin Acupunct Moxibust. 2016;(10):22-5.

43. Zaslawski C, Rogers C, Garvey M, Ryan D, Yang CX, Zhang SP. Strategies to maintain the credibility of sham acupuncture used as a control treatment in clinical trials. J Altern Complement Med. 1997;3(3):257-66. DOI: 10.1089/acm.1997.3.257

44. Chae Y, Um SI, Yi SH, Lee H, Chang DS, Yin CS, et al. Comparison of biomechanical properties between acupuncture and non-penetrating sham needle. Complement Ther Med. 2010 Oct 14;19 Suppl 1:S8-S12. DOI: 10.1016/j.ctim.2010.09.002

45. Chen HP. Introducing a sham acupuncture model design [translated]. Shanghai J Acupunct Moxibust. 2005 Jan;24(1):3-4. DOI: 10.13460/j.issn.10050957.2005.01.002

46. Deng G, Vickers AJ, Yeung S, D'Andrea GM, Xiao H, Heerdt AS, et al. Randomized, controlled trial of acupuncture for the treatment of hot flashes in breast cancer patients. J Clin Oncol. 2007 Dec 10;25(35):5584-90. DOI: 10.1200/JC0.2007.12.0774

47. Dos Santos Maciel LY, Dos Santos Leite PM, Neto ML, Mendonça AC, de Araujo CC, da Hora Santos Souza J, et al. Comparison of the placebo effect between different non-penetrating acupuncture devices and real acupuncture in healthy subjects: a randomized clinical trial. BMC Complement Altern Med. 2016 Dec 15;16(1):518. DOI: 10.1186/s12906-016-1477-2

48. Enblom A, Hammar M, Steineck G, Börjeson S. Can individuals identify if needling was performed with an acupuncture needle or a non-penetrating sham needle? Complement Ther Med. 2008 Oct;16(5):288-94. DOI: 10.1016/j.ctim.2008.02.012

49. Enblom A, Johnsson A, Hammar M, Steineck G, Börjeson S. The nonpenetrating telescopic sham needle may blind patients with different characteristics and experiences when treated by several therapists. Evid Based Complement Alternat Med. 2011;2011:185034. DOI: 10.1155/2011/185034

50. Fink M, Gutenbrunner C, Rollnik J, Karst M. Credibility of a newly designed placebo needle for clinical trials in acupuncture research. Forsch Komplementarmed Klass Naturheilkd. 2001 Dec;8(6):368-72. DOI: 10.1159/000057254

51. Goddard G, Shen Y, Steele B, Springer N. A controlled trial of placebo versus real acupuncture. J Pain. 2005 Apr;6(4):237-42. DOI: 10.1016/j.jpain.2004.12.009

52. Kim S, Lee S, Choi S, Park J, Kim S. Discrimination accuracy between real and sham press needles in the hands. Acupunct Med. 2015 June 4;33(4):293-8. DOI: 10.1136/acupmed-2014-010678

53. Lao L, Bergman S, Hamilton GR, Langenberg P, Berman B. Evaluation of acupuncture for pain control after oral surgery: a placebo-controlled trial. Arch Otolaryngol Head Neck Surg. 1999 May;125(5):567-72. DOI:10.1001/archotol.125.5.567

54. Lee H, Bang H, Kim Y, Park J, Lee S, Lee H, et al. Non-penetrating sham needle, is it an adequate sham control in acupuncture research? Complement Ther Med. 2010 Dec 23;19 Suppl 1:S41-8. DOI: 10.1016/j.ctim.2010.12.002

55. Liang ZH, Xie CC, Li ZP, Zhu XP, Lu AP, Fu WB. Deqi sensation in placebo acupuncture: a crossover study on chinese medicine students. Evid Based Complement Alternat Med. 2013;2013:620671. DOI: 10.1155/2013/620671

56. Liu B, Xu H, Ma R, Mo Q, Yan S, Liu Z. Effect of blinding with a new pragmatic placebo needle: a randomized controlled crossover study. Medicine (Baltimore). 2014 Dec;93(27):1-10. DOI: 10.1097/MD.0000000000000200

57. Smith C, Coyle M, Norman RJ. Influence of acupuncture stimulation on pregnancy rates for women undergoing embryo transfer. Fertil Steril. 2006 May;85(5):1352-8. DOI: 10.1016/j.fertnstert.2005.12.015

58. Smith CA, Crowther CA, Petrucco O, Beilby J, Dent H. Acupuncture to treat primary dysmenorrhea in women: a randomized controlled trial. Evid Based Complement Alternat Med. 2011;2011:612464. DOI: 10.1093/ecam/nep239

59. Takakura N, Yajima H. A placebo acupuncture needle with potential for double blinding - a validation study. Acupunct Med. 2008 Dec;26(4):224-30. DOI: 10.1136/aim.26.4.224

60. Takakura N, Takayama M, Kawase A, Yajima H. Double blinding with a new placebo needle: a validation study on participant blinding. Acupunct Med. 2011 Sep;29(3):203-7. DOI: 10.1136/aim.2010.002857

61. Takakura N, Takayama M, Kawase A, Kaptchuk TJ, Yajima H. Double-blind acupuncture needle: a potential tool to investigate the nature of pain and pleasure. ISRN Pain. 2013;2013:825751. DOI: 10.1155/2013/825751

Page $13 / 20$ 
62. Takayama M, Yajima H, Kawase A, Homma I, Izumizaki M, Takakura N. The potential of double blinding with two placebo acupuncture needles: a randomized controlled pilot-trial. Medicines (Basel). 2014 Dec 30;2(1):11-27. DOI: 10.3390/medicines2010011

63. Tan CW, Christie L, St-Georges V, Telford N. Discrimination of real and sham acupuncture needles using the Park sham device: a preliminary study. Arch Phys Med Rehabil. 2009 Dec;90(12):2141-5. DOI: 10.1016/j.apmr.2009.08.142

64. Tan CW, Sheehan P, Santos D. Discrimination accuracy between real and sham needles using the Park sham device in the upper and lower limbs. Acupunct Med. 2011 Sep;29(3):208-14. DOI: 10.1136/aim.2010.003210

65. Tan CW, Santos D. Contribution of vision, touch, and hearing to the use of sham devices in acupuncture-related studies. J Acupunct Meridian Stud. 2020 Feb;13(1):25-32. DOI: 10.1016/j.jams.2019.12.003

66. To M, Alexander C. The effects of Park sham needles: a pilot study. J Integr Med. 2015 Jan;13(1):20-4. D0I: 10.1016/S2095-4964(15)60153-4

67. Tough EA, White AR, Richards SH, Lord B, Campbell JL. Developing and validating a sham acupuncture needle. Acupunct Med. 2009 Sep;27(3):118-22. DOI: 10.1136/aim.2009.000737

68. Tsukayama H, Yamashita H, Kimura T, Otsuki K. Factors that influence the applicability of sham needle in acupuncture trials: two randomized, singleblind, crossover trials with acupuncture-experienced subjects. Clin J Pain. 2006 May;22(4):346-9. DOI: 10.1097/01.ajp.0000176359.94644.mL

69. Vase L, Baram S, Takakura N, Takayama M, Yajima H, Kawase A, et al. Can acupuncture treatment be double-blinded? An evaluation of double-blind acupuncture treatment of postoperative pain. PLoS One. 2015 Mar 6;10(3):e0119612. DOI: 10.1371/journal.pone.0119612

70. White AR, Eddleston C, Hardie R, Resch KL, Ernst E. A pilot study of acupuncture for tension headache, using a novel placebo. Acupunct Med. 1996 May 1;14(1):11-5. DOI: 10.1136/aim.14.1.11

71. White P, Lewith G, Hopwood V, Prescott P. The placebo needle, is it a valid and convincing placebo for use in acupuncture trials? A randomised, singleblind, cross-over pilot trial. Pain. 2003 Dec;106(3):401-9. DOI: 10.1016/j.pain.2003.08.013

72. White P, Lewith G, Prescott P. Should we recruit patients or healthy volunteers for acupuncture studies of chronic pain? Clin J Pain. 2007 Oct;23(8):714-9. DOI: 10.1097/AJP.0b013e31814da3d2

73. Xie CC, Wen XY, Jiang L, Xie MJ, Fu WB. Validity of the "Streitberger" needle in a Chinese population with acupuncture: a randomized, single-blinded, and crossover pilot study. Evid Based Complement Alternat Med. 2013;2013:251603. DOI: 10.1155/2013/251603

74. A proposed standard international acupuncture nomenclature: report of a WHO scientific group. Switzerland: World Health Organization; 1991.

75. Zhu D, Gao Y, Chang J, Kong J. Placebo acupuncture devices: considerations for acupuncture research. Evid Based Complement Alternat Med. 2013;2013:628907. DOI: 10.1155/2013/628907

76. Zhang HW, Tang JL. The design and selection of placebo acupuncture needles in acupuncture clinical trials [translated]. Chin J Integrat Trad West Med. 2003 Apr;23(4):247-50.

77. Witt CM. Clinical research on acupuncture - concepts and guidance on efficacy and effectiveness research. Chin J Integr Med. 2011 Mar;17(3):166-72. DOI: $10.1007 / \mathrm{s} 11655-011-0662-x$

78. Chen H, Yang M, Ning Z, Lam WL, Zhao YK, Yeung WF, et al. A Guideline for Randomized Controlled Trials of Acupuncture. Am J Chin Med. 2019;47(1):118. DOI:10.1142/S0192415X19500010

\section{Figures}




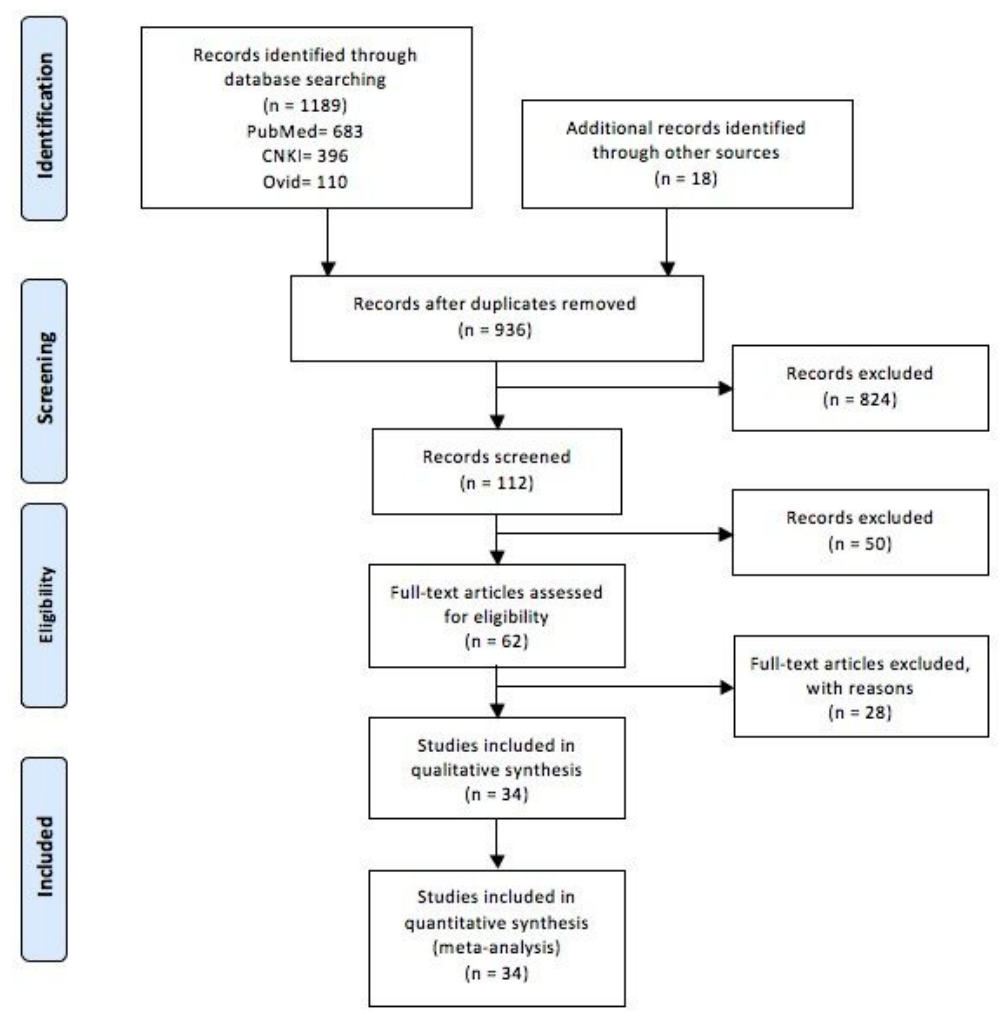

Figure 1

PRISMA Flow Diagram

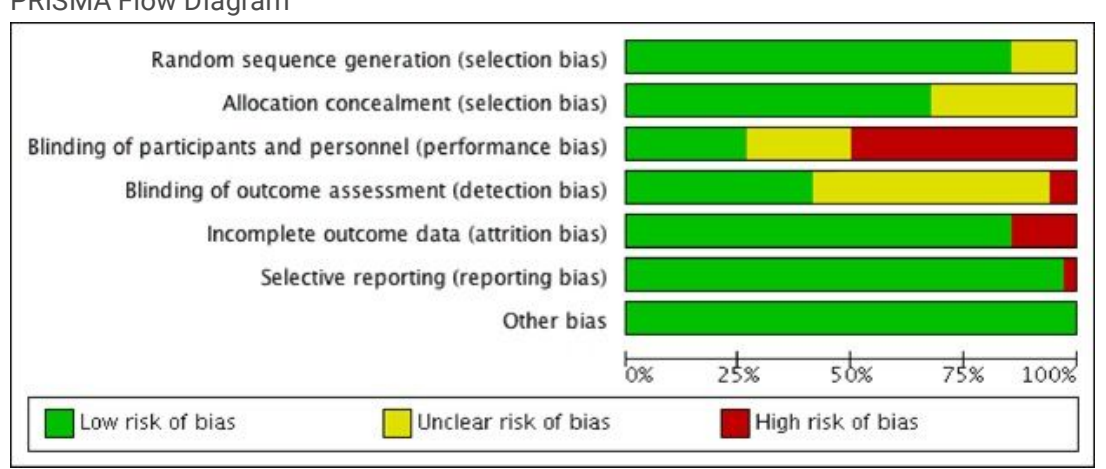

\section{Figure 2}

Risk of Bias Graph 


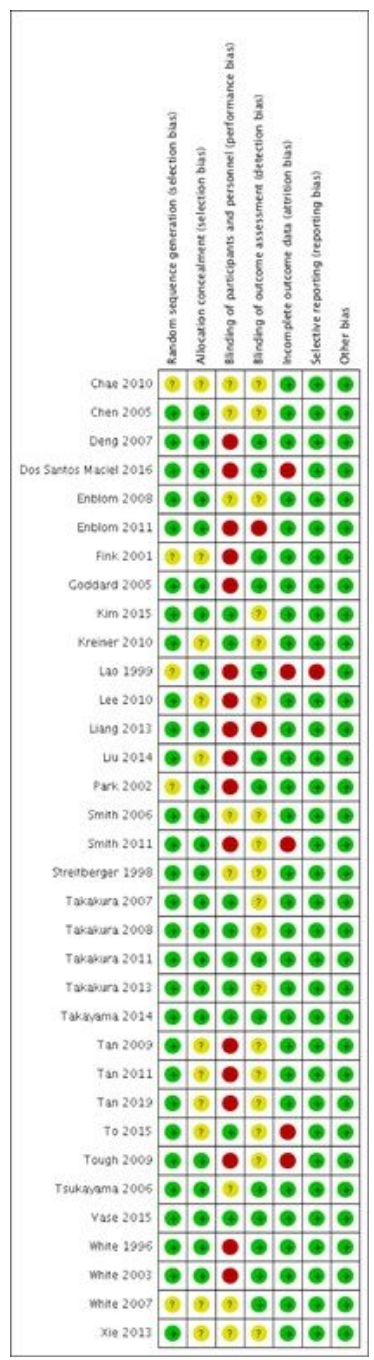

Figure 3

Risk of Bias Summary

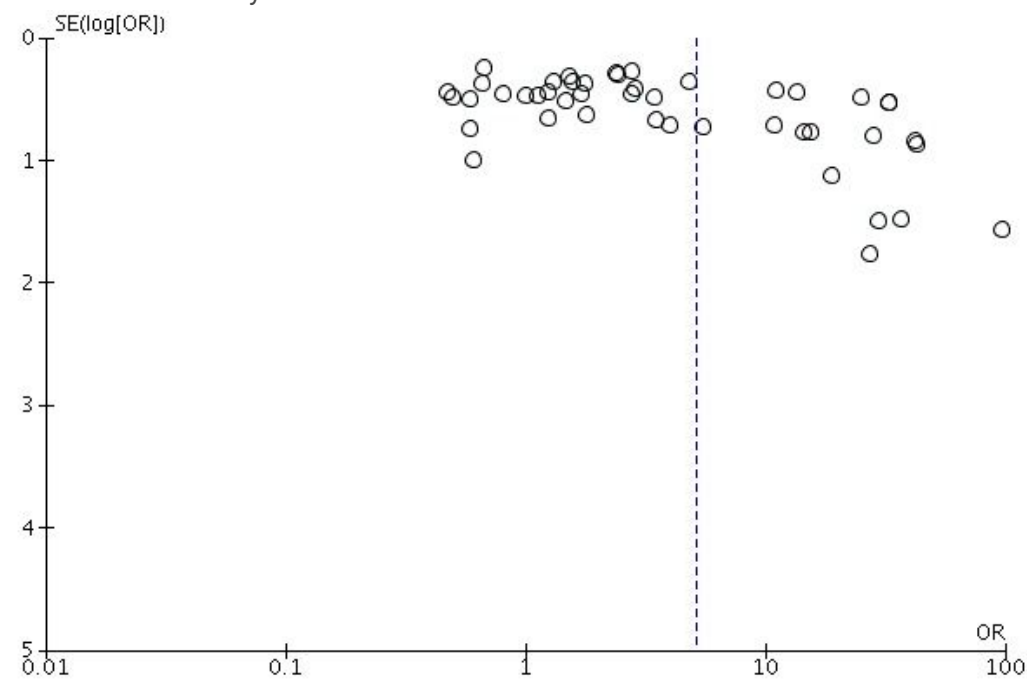

Figure 4

Funnel Plot 


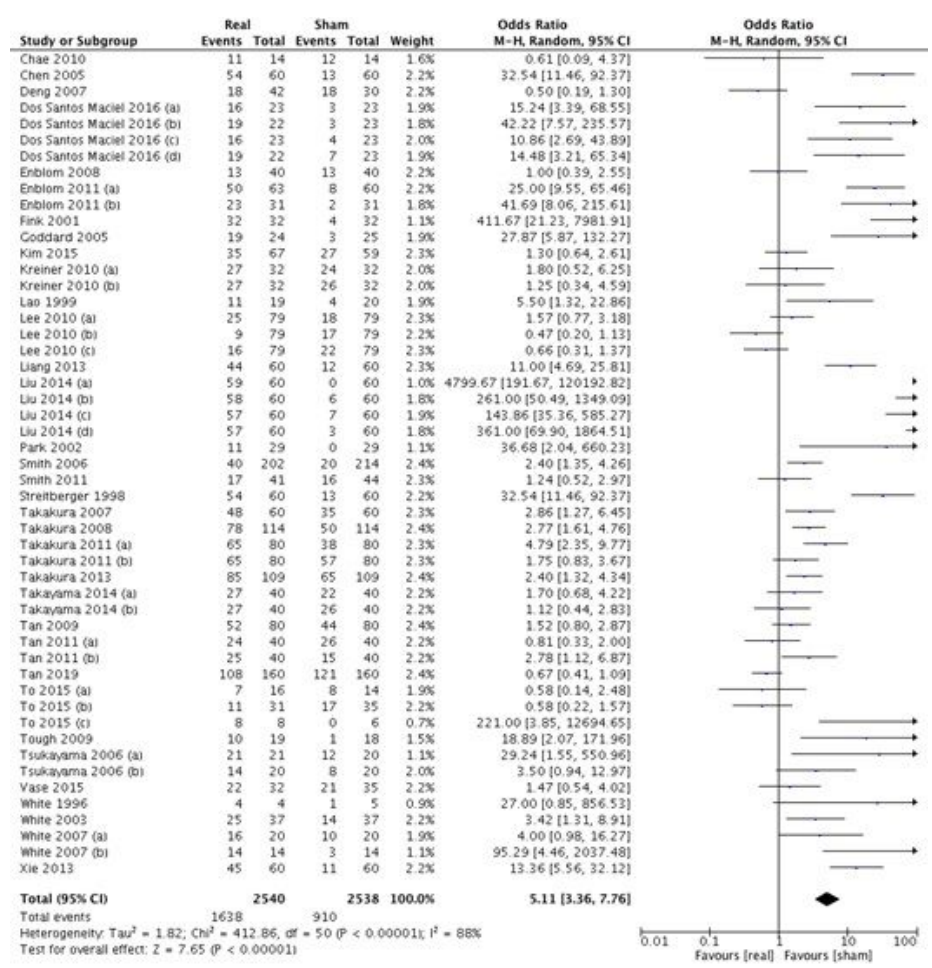

Figure 5

Overall Blinding Effectiveness of Sham Acupuncture Devices

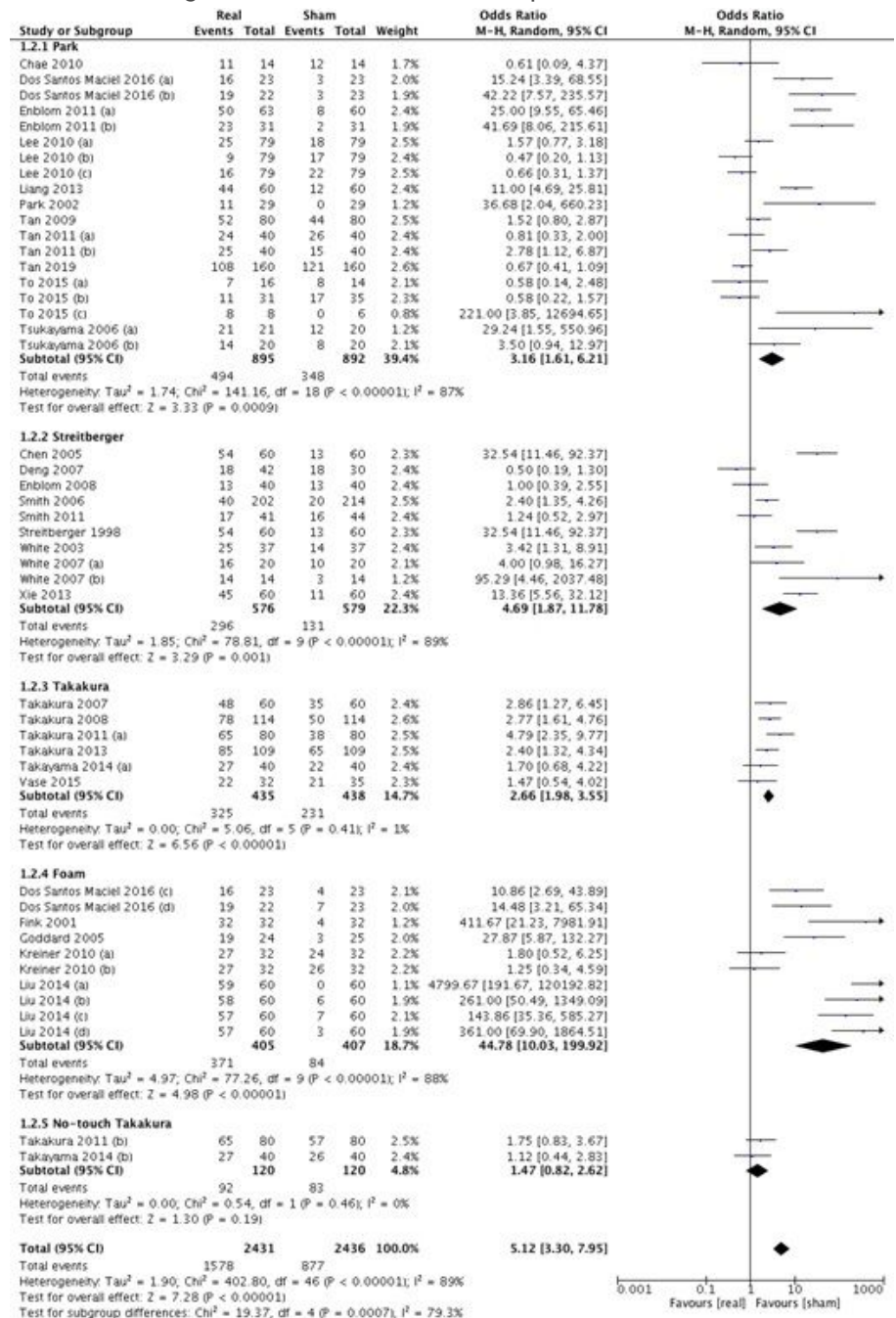


Figure 6

Comparison between Different Types of Sham Acupuncture Devices

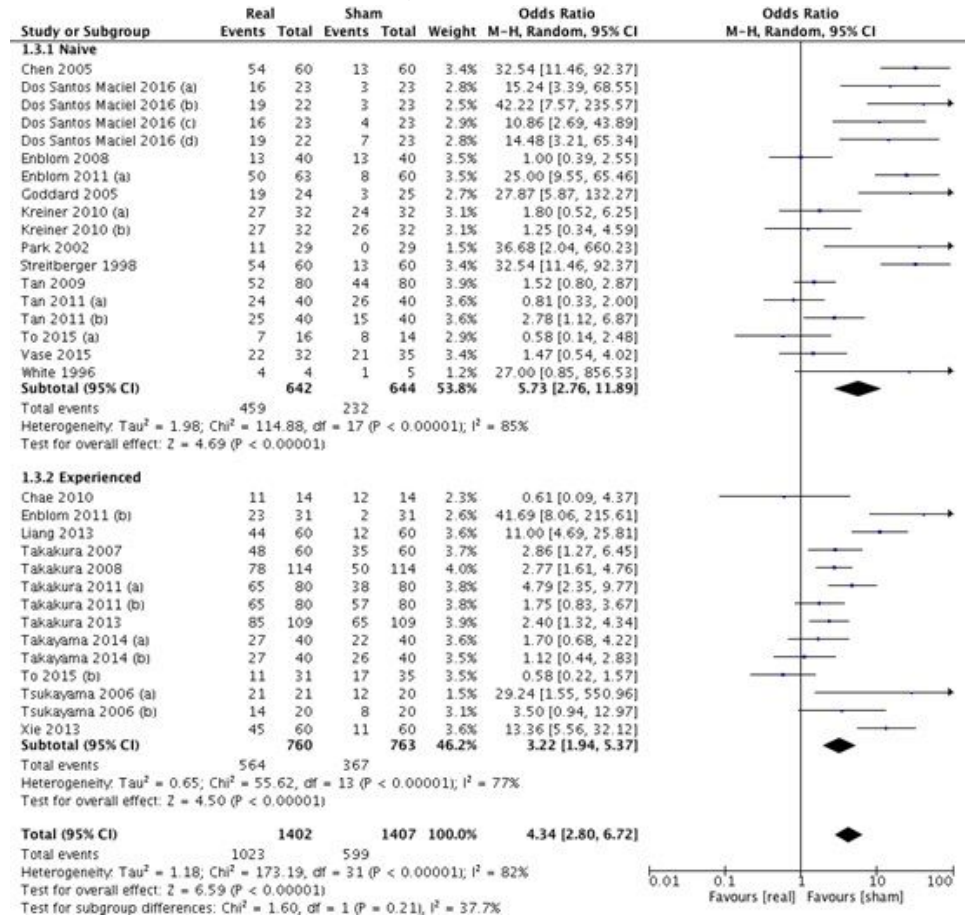

\section{Figure 7}

Blinding Effectiveness Based on Acupuncture Experience

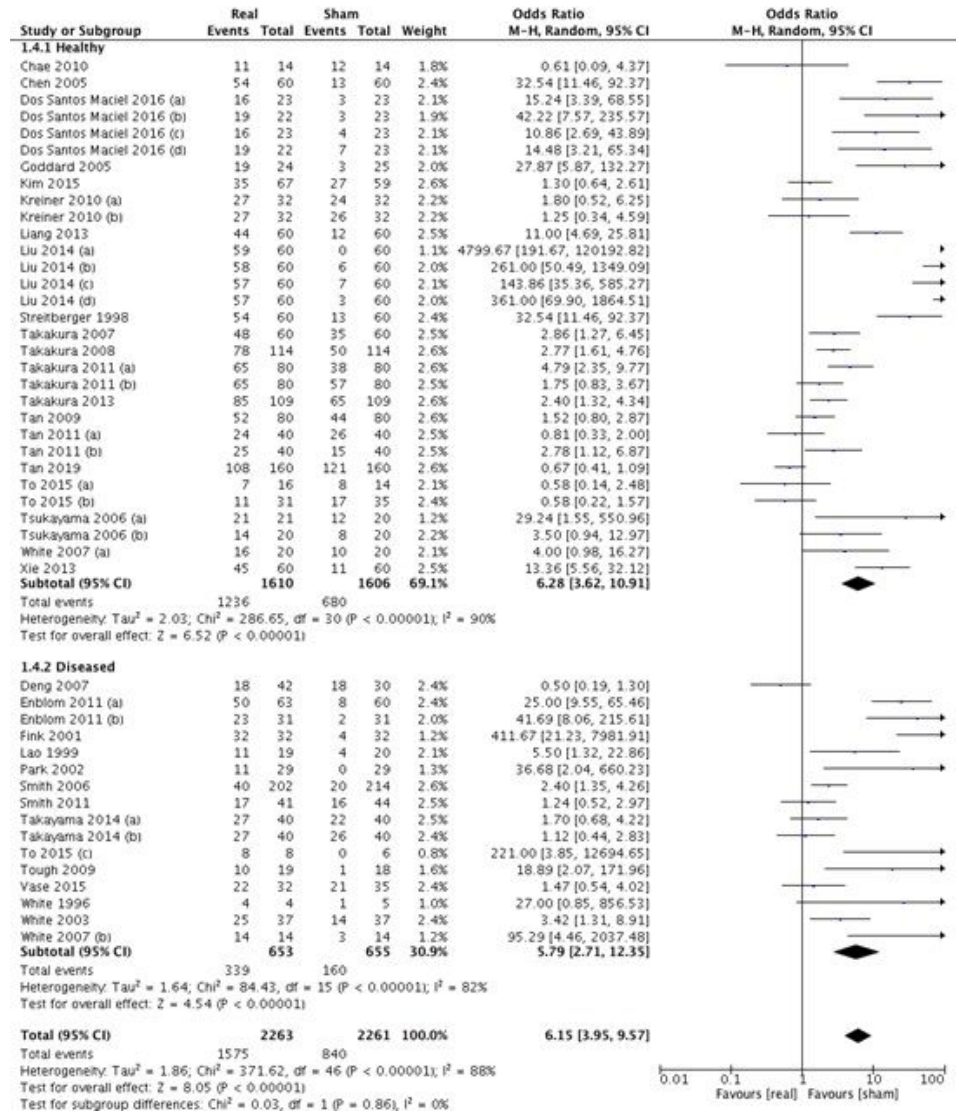

Figure 8

Blinding Effectiveness Based on Health Status 


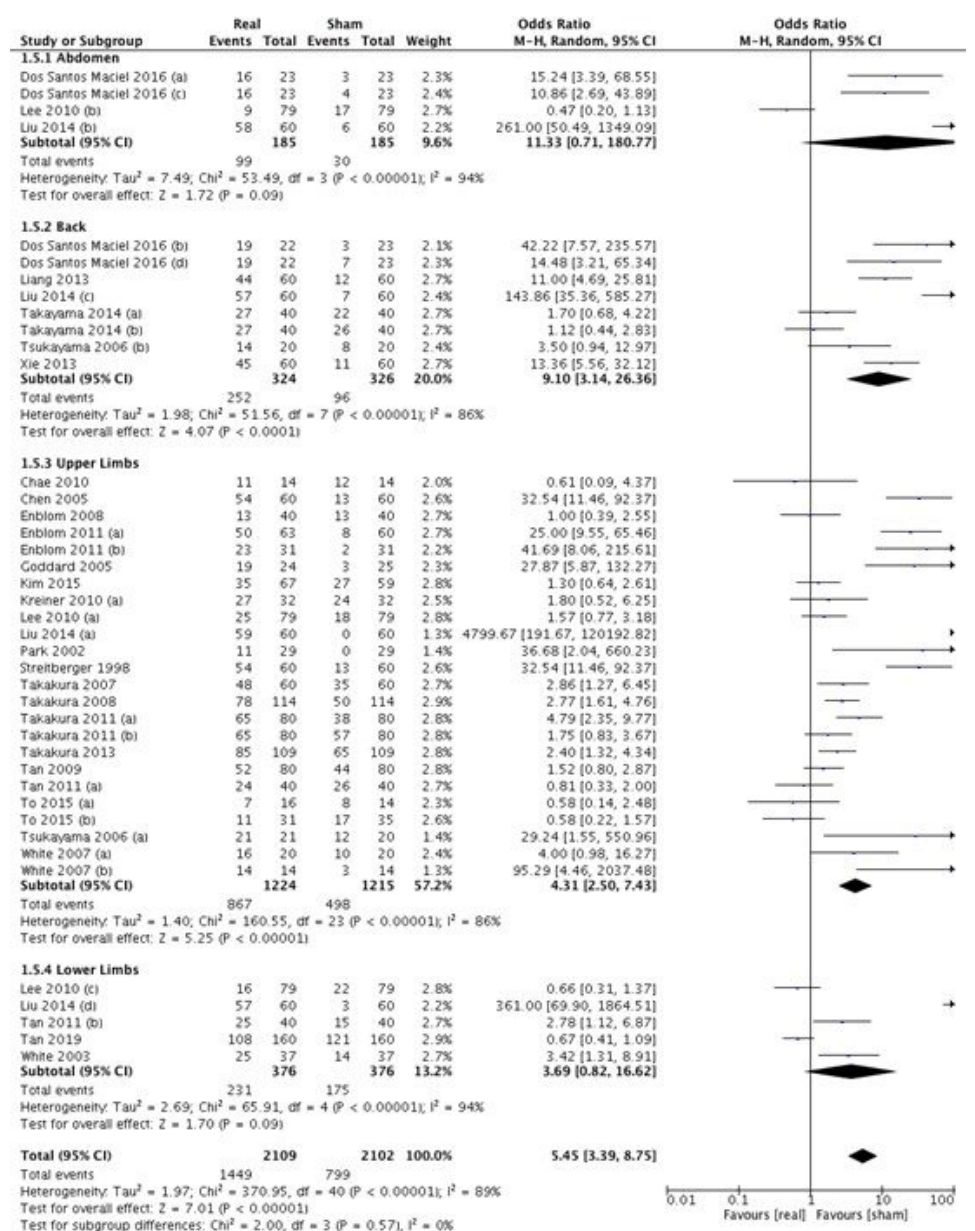

\section{Figure 9}

Blinding Effectiveness Based on the Locations of Acupoints

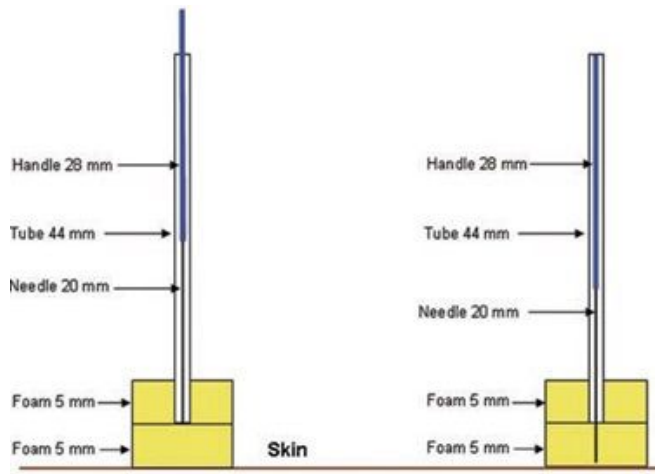

Figure 10

Foam Sham Acupuncture Device10

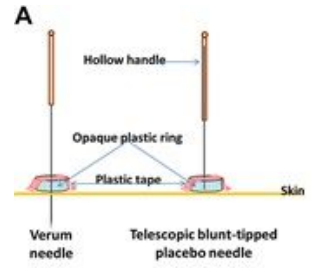

The Streitberger device

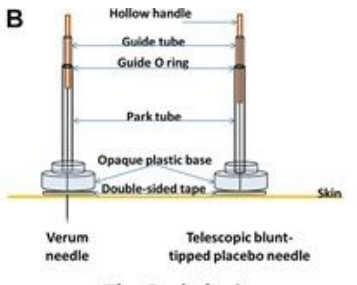

The Park device

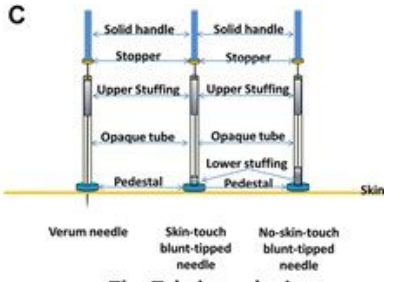

The Takakura device

\section{Figure 11}

Three Types of Non-penetrating Sham Acupuncture Devices11 


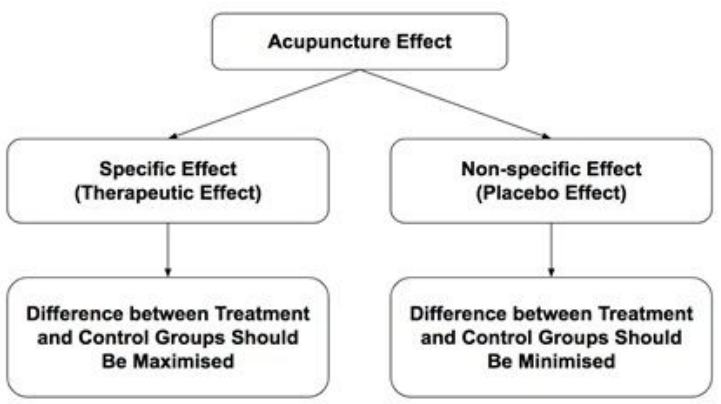

Figure 12

Acupuncture Effect in Sham Acupuncture Controlled Clinical Trials 